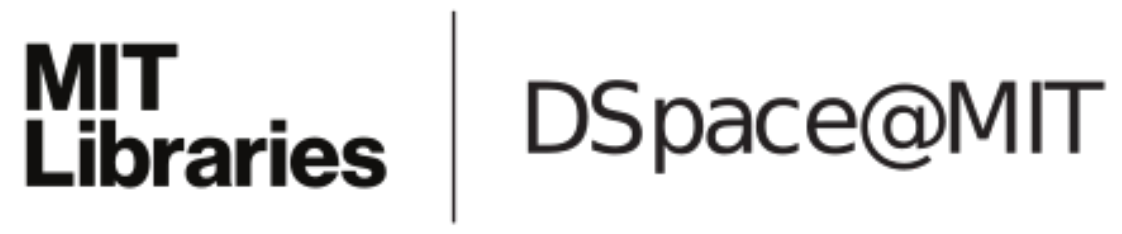

\author{
MIT Open Access Articles
}

A space-time certified reduced basis method for Burgers' equation

The MIT Faculty has made this article openly available. Please share how this access benefits you. Your story matters.

Citation: Yano, Masayuki, Anthony T. Patera, and Karsten Urban. "A Space-Time HpInterpolation-Based Certified Reduced Basis Method for Burgers' Equation." Math. Models Methods Appl. Sci. 24, no. 09 (August 2014): 1903-1935.

As Published: http://dx.doi.org/10.1142/S0218202514500110

Publisher: World Scientific

Persistent URL: http://hdl.handle.net/1721.1/97700

Version: Original manuscript: author's manuscript prior to formal peer review

Terms of use: Creative Commons Attribution-Noncommercial-Share Alike 


\section{A Space-Time Certified Reduced Basis Method for Burgers' Equation}

Masayuki Yano, Anthony T. Patera und Karsten Urban

Preprint Series: 2012 - 09

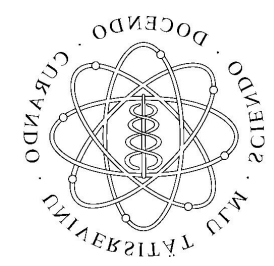

Fakultät für Mathematik und Wirtschaftswissenschaften UNIVERSITÄT ULM 


\title{
A SPACE-TIME CERTIFIED REDUCED BASIS METHOD FOR BURGERS' EQUATION
}

\author{
MASAYUKI YANO \\ Department of Mechanical Engineering, Massachusetts Institute of Technology, \\ Cambridge, MA 02139, USA \\ myano@mit.edu \\ ANTHONY T. PATERA \\ Department of Mechanical Engineering, Massachusetts Institute of Technology, \\ Cambridge, MA 02139, USA \\ patera@mit.edu \\ KARSTEN URBAN \\ Institute for Numerial Mathematics, Ulm University, \\ Helmholtzstrasse 18, 89081, Ulm, Germany \\ karsten.urban@uni-ulm.de \\ Received (Day Month Year) \\ Revised (Day Month Year) \\ Communicated by (xxxxxxxxxx)
}

\begin{abstract}
We present a space-time certified reduced basis method for Burgers' equation over the spatial interval $(0,1)$ and the temporal interval $(0, T]$ parametrized with respect to the Peclet number. We first introduce a Petrov-Galerkin space-time finite element discretization, which enjoys a favorable inf-sup constant that decreases slowly with Peclet number and final time $T$. We then consider an $h p$ interpolation-based space-time reduced basis approximation and associated Brezzi-Rappaz-Raviart a posteriori error bounds. We detail computational procedures that permit offline-online decomposition for the three key ingredients of the error bounds: the dual norm of the residual, a lower bound for the inf-sup constant, and the space-time Sobolev embedding constant. Numerical results demonstrate that our space-time formulation provides improved stability constants compared to classical $L^{2}$-error estimates; the error bounds remain sharp over a wide range of Peclet numbers and long integration times $T$, unlike the exponentially growing estimate of the classical formulation for high Peclet number cases.
\end{abstract}

Keywords: space-time variational formulation, parametrized parabolic equations, quadratic noninearity, reduced basis, Brezzi-Rappaz-Raviart theory, a posteriori error bounds, inf-sup constant, Burgers' equation

AMS Subject Classification: 22E46, 53C $35,57 \mathrm{~S} 20$ 


\section{Introduction}

In this paper, we develop a certified reduced basis method for the parametrized unsteady Burgers' equation. Classically, parametrized parabolic partially differential equations (PDEs) are treated by collecting solution snapshots in the parametertime space and by constructing the reduced basis space using the proper orthogonal decomposition of the snapshots. ${ }^{4,5,9,7}$ Such a formulation enables rapid approximation of parametrized PDEs by an offline-online computational decomposition, and the reduced basis solution converges exponentially to the truth finite element for sufficiently regular problems. However, the quality of the associated $L^{2}$-in-time $a$ posteriori error bound relies on the coercivity of the spatial operator. If the spatial operator is non-coercive, the formulation suffers from exponential temporal instability, producing error bounds that grow exponentially in time, rendering the bounds meaningless for long-time integration. In particular, limited applicability of the classical a posteriori error bounding technique to unsteady Burgers' and Boussinesq equations are documented by Nguyen et al. ${ }^{9}$ and Knezevic et al. ${ }^{7}$, respectively.

In order to overcome the instability of the classical $L^{2}$-in-time error-bound formulation, we follow the space-time approach recently devised by Urban and Patera, ${ }^{13,12}$; we consider a space-time variational and corresponding finite element formulation that produces a favorable inf-sup stability constant and then incorporate the space-time truth within a space-time reduced basis approach. The approach is inspired by the recent work on the space-time Petrov-Galerkin formulation by Schwab and Stevenson ${ }^{11}$.

The main contribution of this work is twofold. First is the application of the space-time finite-element and reduced-basis approach to the unsteady Burgers' equation with quadratic nonlinearity. The formulation results in Crank-Nicolson-like time-marching procedure but benefits from full space-time variational interpretation and favorable inf-sup stability constant. The second contribution is the application of the Brezzi-Rappaz-Raviart theory to the space-time formulation to construct an error bound for the quadratic nonlinearity. Particular attention is given to the development of an efficient computation procedure that permits offline-online decomposition for the three key ingredients of the theory: the dual norm of the residual; an inf-sup lower bound, and the Sobolev embedding constant.

This paper is organized as follows. Section 2 reviews the spaces and forms used throughout this paper and introduces a space-time Petrov-Galerkin variational and finite element formulation of the Burgers' equation. Section 3 first presents an $h p$ interpolation-based reduced basis approximation and then an associated a posteriori error estimate based on the Brezzi-Rappaz-Raviart theory. The section details the calculation of the dual-norm of the residual, an inf-sup lower bound, and the space-time Sobolev embedding constant, paying particular attention to the offlineonline computational decomposition. Finally, Section 4 considers two examples of Burgers' problems and demonstrates that the new space-time error bound provides a meaningful error estimate even for noncoercive cases for which the classical esti- 
mate fails. We also demonstrate that the $h p$ interpolation method provides certified solutions over a wide range of parameters using a reasonable number of points. Although we consider single-parameter, one-dimensional Burgers' equation in order to simplify the presentation and facilitate numerical tests, the method extends to multi-dimensional incompressible Navier-Stokes equations and several parameters as will be considered in future work.

\section{Truth Solution}

\subsection{Governing Equation}

This work considers a parametrized, unsteady, one-dimensional Burgers' equation of the form

$$
\frac{\partial \tilde{u}}{\partial \tilde{t}}+\frac{\partial}{\partial x}\left(\frac{1}{2} \tilde{u}^{2}\right)-\frac{1}{\operatorname{Pe}} \frac{\partial^{2} \tilde{u}}{\partial x^{2}}=g(x), \quad x \in \Omega, \tilde{t} \in \tilde{I},
$$

where $\tilde{u}$ is the state variable, Pe is the Peclet number, $g$ is the forcing term, $\Omega \equiv(0,1)$ is the unit one-dimensional domain, and $I \equiv(0, \tilde{T}]$ is the temporal interval with $\tilde{T}$ denoting the final time of interest. We impose homogeneous Dirichlet boundary conditions,

$$
\tilde{u}(0, t)=\tilde{u}(1, t)=0, \quad \forall t \in I,
$$

and set the initial condition to

$$
\tilde{u}(x, 0)=0, \quad \forall x \in \Omega .
$$

Setting $t=\tilde{t} / \mathrm{Pe}$ and $u=\mathrm{Pe} \cdot \tilde{u}$, Eq. (2.1) simplifies to

$$
\frac{\partial u}{\partial t}+\frac{\partial}{\partial x}\left(\frac{1}{2} u^{2}\right)-\frac{\partial^{2} u}{\partial x^{2}}=\mathrm{Pe}^{2} \cdot g(x), \quad x \in \Omega, t \in I .
$$

Note that the transformation makes the left hand side of the equation independent of the parameter Pe. The homogeneous boundary conditions and the initial condition are unaltered by the transformation. Moreover, note that $T=\mathcal{O}(1)$ represents a long time integration from $t=0$ to $\tilde{T}=\mathcal{O}(\mathrm{Pe})$ based on the convection time scale. From hereon, we will exclusively work with this transformed form of the Burgers' equation, Eq. (2.2).

\subsection{Spaces and Forms}

Let us now define a few spaces and forms that are used throughout this paper. ${ }^{10}$ The standard $L^{2}(D)$ Hilbert space over an arbitrary domain $D$ is equipped with an inner product $(\psi, \phi)_{L^{2}(D)} \equiv \int_{\Omega} \psi \phi d x$ and a norm $\|\psi\|_{L^{2}(D)} \equiv \sqrt{(\psi, \psi)_{L^{2}(D)}}$. The $H^{1}(D)$ space is equipped with an inner product $(\psi, \phi)_{H^{1}(D)} \equiv \int_{\Omega} \nabla \psi \cdot \nabla \phi d x$ and an inner product $\|\psi\|_{H^{1}(D)} \equiv \sqrt{(\psi, \psi)_{H^{1}(D)}}$. We also introduce a space of trace-free functions $H_{0}^{1}(D) \equiv\left\{v \in H^{1}(D):\left.v\right|_{\partial D}=0\right\}$ equipped with the same inner product and norm as $H^{1}(D)$. We define Gelfand triple $\left(V, H, V^{\prime}\right)$ and associated duality 
paring $\langle\cdot, \cdot\rangle_{V^{\prime} \times V}$ where, in our context, $V \equiv H_{0}^{1}(\Omega)$ and $H \equiv L^{2}(\Omega)$. Here the norm of $\ell \in V^{\prime}$ is defined by $\|\ell\|_{V^{\prime}} \equiv \frac{\langle\ell, \phi\rangle_{V^{\prime} \times V}}{\|\phi\|_{V}}$, which is identical to $\|R \ell\|_{V}$ where the Riesz operator $R: V^{\prime} \rightarrow V$ satisfies $(R \ell, \phi)_{V}=\langle\ell, \phi\rangle_{V^{\prime} \times V}, \forall \ell \in V^{\prime}, \forall \phi \in V$.

Let us now define space-time spaces, which play key roles in our space-time formulation. The space $L^{2}(I ; V)$ is equipped with an inner product

$$
(w, v)_{L^{2}(I ; V)} \equiv \int_{I}(w(t), v(t))_{V} d t
$$

and a norm $\|w\|_{L^{2}(I ; V)} \equiv \sqrt{(w, w)_{L^{2}(I ; V)}}$. The dual space $L^{2}\left(I ; V^{\prime}\right)$ is equipped with an inner product

$$
(w, v)_{L^{2}\left(I ; V^{\prime}\right)} \equiv \int_{I}(R w(t), R v(t))_{V} d t
$$

and a norm $\|w\|_{L^{2}\left(I ; V^{\prime}\right)} \equiv \sqrt{(w, w)_{L^{2}\left(I ; V^{\prime}\right)}}$, where $R: V^{\prime} \rightarrow V$ is the aforementioned Riesz operator. The space $H_{(0)}^{1}\left(I ; V^{\prime}\right)$ is equipped with an inner product $(w, v)_{H^{1}\left(I ; V^{\prime}\right)} \equiv(\dot{w}, \dot{v})_{L^{2}\left(I ; V^{\prime}\right)}$ and a norm $\|w\|_{H^{1}\left(I ; V^{\prime}\right)} \equiv \sqrt{(w, w)_{H^{1}\left(I ; V^{\prime}\right)}}$ and consists of functions $\left\{w:\|w\|_{H^{1}\left(I ; V^{\prime}\right)}<\infty, w(0)=0\right\}$; here $\dot{w} \equiv \frac{\partial w}{\partial t}$ denotes the temporal derivative of $w$. The trial space for our space-time Burgers' formulation is

$$
\mathcal{X} \equiv L^{2}(I ; V) \cap H_{(0)}^{1}\left(I ; V^{\prime}\right)
$$

equipped with an inner product

$$
(w, v)_{\mathcal{X}} \equiv(w, v)_{H^{1}\left(I ; V^{\prime}\right)}+(w, v)_{L^{2}(I ; V)}
$$

and a norm $\|w\|_{\mathcal{X}} \equiv \sqrt{(w, w)_{\mathcal{X}}}$. Note that $\|w\|_{\mathcal{X}}^{2}=\|w\|_{H^{1}\left(I ; V^{\prime}\right)}^{2}+\|w\|_{L^{2}(I ; V)}^{2} \cdot{ }^{\text {a }}$ The test space is $\mathcal{Y} \equiv L^{2}(I ; V)$.

Having defined spaces, we are ready to express the governing equation, Eq. (2.2), in a weak form. We may seek a solution to the Burgers' equation expressed in a semi-weak form: find $\psi \in C_{(0)}^{0}\left(I ; L^{2}(\Omega)\right) \cap L^{2}(I ; V)$ such that ${ }^{10}$

$$
(\dot{\psi}(t), \phi)_{H}+a(\psi(t), \phi)+b(\psi(t), \psi(t), \phi)=f(\phi ; \mathrm{Pe}), \quad \forall \phi \in V, \forall t \in I,
$$

where $C^{p}$ is the space of functions with continuous $p$-th derivative, and $C_{(0)}^{p}$ is the subspace of $C^{p}$ that consists of functions satisfying the zero initial condition. The bilinear form $a(\cdot, \cdot)$, the trilinear form $b(\cdot, \cdot, \cdot)$, and the parametrized linear form $f(\cdot ; \mathrm{Pe})$ are given by

$$
\begin{aligned}
a(\psi, \phi) & \equiv \int_{\Omega} \frac{\partial \psi}{\partial x} \frac{\partial \phi}{\partial x} d x, \quad \forall \psi, \phi \in V \\
b(\psi, \zeta, \phi) & \equiv-\frac{1}{2} \int_{\Omega} \psi \zeta \frac{d \phi}{d x} d x, \quad \forall \psi, \zeta, \phi \in V \\
f(\phi ; \mathrm{Pe}) & \equiv \mathrm{Pe}^{2} \cdot\langle g, \phi\rangle_{V^{\prime} \times V} \quad \forall \phi \in V .
\end{aligned}
$$

a The $\mathcal{X}$-norm used in this work is slightly weaker than that used in Urban and Patera ${ }^{13,12}$ that includes the terminal condition, $\|w(T)\|_{H}^{2}$. 
Note that the trilinear form $b(\cdot, \cdot, \cdot)$ is symmetric in the first two arguments. By choosing $\mu=\mathrm{Pe}^{2}$, we can express the linear form as a linear function of the parameter $\mu$, i.e.

$$
f(\phi ; \mu) \equiv \mu \cdot\langle g, \phi\rangle_{V^{\prime} \times V} .
$$

Thus, our linear form permits so-called affine decomposition with respect to the parameter $\mu$. (We note that the certified reduced-basis formulation presented in this work readily treats any $f$ that is affine in a function of parameter $\mu$, though the work is presented for the simple single-parameter case above.)

More generally, we can seek the solution to the Burgers' equation in the spacetime space $\mathcal{X}$. Namely, a space-time weak statement reads: Find $u \in \mathcal{X}$ such that

$$
\mathcal{G}(u, v ; \mu)=0, \quad \forall v \in \mathcal{Y},
$$

where the semilinear form $\mathcal{G}(\cdot, \cdot ; \mu)$ is given by

$$
\mathcal{G}(w, v ; \mu)=\mathcal{M}(\dot{w}, v)+\mathcal{A}(w, v)+\mathcal{B}(w, w, v)-\mathcal{F}(v ; \mu), \quad \forall w \in \mathcal{X}, \forall v \in \mathcal{Y},
$$

with the space-time forms

$$
\begin{aligned}
\mathcal{M}(\dot{w}, v) & \equiv \int_{I}\langle\dot{w}(t), v(t)\rangle_{V^{\prime} \times V} d t, \quad \forall w \in \mathcal{X}, \forall v \in \mathcal{Y}, \\
\mathcal{A}(w, v) & \equiv \int_{I} a(w(t), v(t)) d t, \quad \forall w \in \mathcal{X}, \forall v \in \mathcal{Y}, \\
\mathcal{B}(w, z, v) & \equiv \int_{I} b(w(t), z(t), v(t)) d t, \quad \forall w \in \mathcal{X}, \forall v \in \mathcal{Y}, \\
\mathcal{F}(v ; \mu) & \equiv \mu \cdot \int_{I}\langle g, v(t)\rangle_{V^{\prime} \times V} d t, \quad \forall v \in \mathcal{Y} .
\end{aligned}
$$

Note that the trilinear form $\mathcal{B}(\cdot, \cdot, \cdot)$ inherits the symmetry with respect to the first two arguments. Furthermore, we will denote the Fréchet derivative bilinear form associated with $\mathcal{G}$ by $\partial \mathcal{G}$, i.e.

$$
\partial \mathcal{G}(w, z, v)=\mathcal{M}(\dot{w}, v)+\mathcal{A}(w, v)+2 \mathcal{B}(w, z, v), \quad \forall w, z \in \mathcal{X}, \forall v \in \mathcal{Y},
$$

where $z \in \mathcal{X}$ is the linearization point.

Let us note a few important properties of our unsteady Burgers' problem. First, our space-time linear form $\mathcal{F}$ permits trivial affine-decomposition, i.e. $\mathcal{F}(v ; \mu)=$ $\mu \mathcal{F}_{0}(v)$ where $\mathcal{F}_{0}=\int_{I}\langle g, v(t)\rangle_{V^{\prime} \times V} d t$. Second, our trilinear form is bounded by

$$
|\mathcal{B}(w, z, v)| \equiv\left|\int_{I} \int_{\Omega}-\frac{1}{2} w z \frac{\partial v}{\partial x} d x d t\right| \leq \frac{1}{2} \rho^{2}\|w\|_{\mathcal{X}}\|z\|_{\mathcal{X}}\|v\|_{\mathcal{Y}}, \quad \forall w, z \in \mathcal{X}, \forall v \in \mathcal{Y},
$$

where $\rho$ is the $L^{4}-\mathcal{X}$ embedding constant

$$
\rho \equiv \sup _{w \in \mathcal{X}} \frac{\|w\|_{L^{4}\left(I ; L^{4}(\Omega)\right)}}{\|w\|_{\mathcal{X}}}
$$


Recall that the $L^{p}$ norm is defined as $\|w\|_{L^{p}\left(I ; L^{p}(\Omega)\right)} \equiv\left(\int_{I} \int_{\Omega} w^{p} d x d t\right)^{1 / p}$. This second property plays an important role in applying the Brezzi-Rappaz-Raviart theory to construct an a posteriori error bound. Although we consider only Burgers' equation in this paper, we can readily extend the formulation to any quadratically nonlinear equation which satisfies suitable hypotheses on the forms, as implicitly verified above for Burgers'. (We can also consider non-time-invariant operators subject to the usual affine restrictions.)

\subsection{Petrov-Galerkin Finite Element Approximation}

To find a discrete approximation to the true solution $u \in \mathcal{X}$, let us introduce finite dimensional subspaces $\mathcal{X}_{\delta} \subset \mathcal{X}$ and $\mathcal{Y}_{\delta} \subset \mathcal{Y}$. The notation used in this section closely follows that of Urban and Patera. ${ }^{12}$ We denote the triangulations of the temporal interval and spatial domain by $\mathcal{T}_{\Delta t}^{\text {time }}$ and $\mathcal{T}_{h}^{\text {space }}$, respectively. In particular, $\mathcal{T}_{\Delta t}^{\text {time }}$ consists of non-overlapping intervals $I^{k}=\left(t^{k-1}, t^{k}\right], k=1, \ldots, K$, with $t^{0}=0$ and $t^{K}=T$; here $\max _{k}\left(\left|I^{k}\right|\right) / T \leq \Delta t$ and the family $\left\{\mathcal{T}_{\Delta t}\right\}_{\Delta t \in(0,1]}$ is assumed to be quasi-uniform. Similarly, $\mathcal{T}_{h}^{\text {space }}$ consists of $\mathcal{N}+1$ elements with $\max _{\kappa \in \mathcal{T}_{h}} \operatorname{diam}(\kappa) \leq$ $h$, belonging to a quasi-uniform family of meshes. Let us introduce a temporal trial space $S_{\Delta t}$, a temporal test space $Q_{\Delta t}$, and a spatial approximation space $V_{h}$ defined by

$$
\begin{aligned}
S_{\Delta t} & \equiv\left\{v \in H_{(0)}^{1}(I):\left.v\right|_{I^{k}} \in \mathbb{P}^{1}\left(I^{k}\right), k=1, \ldots, K\right\}, \\
Q_{\Delta t} & \equiv\left\{v \in L^{2}(I):\left.v\right|_{I^{k}} \in \mathbb{P}^{0}\left(I^{k}\right), k=1, \ldots, K\right\}, \\
V_{h} & \equiv\left\{v \in H_{0}^{1}(\Omega):\left.v\right|_{\kappa} \in \mathbb{P}^{1}(\kappa), \kappa \in \mathcal{T}_{h}\right\} .
\end{aligned}
$$

Our space-time finite element trial and test spaces are given by

$$
\mathcal{X}_{\delta}=S_{\Delta t} \otimes V_{h} \quad \text { and } \quad \mathcal{Y}_{\delta}=Q_{\Delta t} \otimes V_{h},
$$

respectively, where $\delta=(\Delta t, h)$ is the characteristic scale of our space-time discretization. Furthermore, we equip the space $\mathcal{X}_{\delta}$ with a mesh-dependent inner product

$$
(w, v)_{\mathcal{X}_{\delta}} \equiv(w, v)_{H^{1}\left(I ; V^{\prime}\right)}+(\bar{w}, \bar{v})_{L^{2}(I ; V)} .
$$

Here $\bar{w} \in \mathcal{Y}_{\delta}$ is a temporally piecewise constant function whose value over $I^{k}$ is the temporal average of the function $w \in \mathcal{X}_{\delta}$, i.e.

$$
\bar{w}^{k} \equiv \frac{1}{\Delta t^{k}} \int_{I^{k}} w d t, \quad k=1, \ldots, K .
$$

We also introduce an associated induced norm $\|w\|_{\mathcal{X}_{\delta}}^{2}=(w, w) \mathcal{X}_{\delta}$. The choice of this mesh-dependent norm is motivated by the fact that, with a slight modification to $\|w\|_{\mathcal{X}_{\delta}}^{2}+\|w(T)\|_{H}^{2}$, the norm provides the unity inf-sup and continuity constant for the Petrov-Galerkin finite element discretization of the heat equation. ${ }^{13,12}$ The space $\mathcal{Y}_{\delta}$ is equipped with the same inner product and the norm as the space $\mathcal{Y}$. 
Our discrete approximation to Burgers' equation, Eq. (2.3), is given by: Find $u_{\delta} \in \mathcal{X}_{\delta}$ such that

$$
\mathcal{G}\left(u_{\delta}, v_{\delta} ; \mu\right)=0, \quad \forall v_{\delta} \in \mathcal{Y}_{\delta} .
$$

The well-posedness of the space-time finite element formulation will be verified $a$ posteriori using the Brezzi-Rappaz-Raviart theory. The temporal integration required for the evaluation of the source term $\mathcal{F}$ is performed using the trapezoidal rule.

\subsection{Algebraic Forms and Time-Marching Interpretation}

In this subsection, we construct algebraic forms of temporal, spatial, and space-time operators required for computing our finite element approximation, various norms, and evaluating inf-sup constants. In addition, we demonstrate that our PetrovGalerkin finite element formulation can in fact be written as a time-stepping scheme for a particular set of trial and test basis functions.

Throughout this section, we will use standard hat-functions $\sigma^{k}$ with the node at $t^{k}, k=1, \ldots, K$, as our basis functions for $S_{\Delta t}$; note that $\operatorname{supp}\left(\sigma^{k}\right)=I^{k} \cup I^{k+1}$ (except for $\sigma^{K}$, which is truncated to have $\operatorname{supp}\left(\sigma^{K}\right)=I^{K}$ ). We further choose characteristic functions $\tau^{k}=\chi_{I^{k}}$ as our basis functions for $Q_{\Delta t}$. Finally, let $\phi_{i}, i=$ $1, \ldots, \mathcal{N}$, be standard hat-functions for $V_{h}$. With the specified basis, we can express a space-time trial function $w_{\delta} \in \mathcal{X}_{\delta}$ in terms of basis coefficients $\left\{w_{i}^{k}\right\}_{i=1, \ldots, \mathcal{N}}^{k=1, \ldots, K}$ as $w_{\delta}=\sum_{k=1}^{K} \sum_{i=1}^{\mathcal{N}} w_{i}^{k} \sigma^{k} \otimes \phi_{i}$; similarly a trial function $v_{\delta} \in \mathcal{Y}_{\delta}$ may be expressed as $v_{\delta}=\sum_{k=1}^{K} \sum_{i=1}^{\mathcal{N}} v_{i}^{k} \tau^{k} \otimes \phi_{i}$. The following sections introduce temporal, spatial, and space-time matrices and their explicit expressions that facilitate evaluation of the residual, norms, and inf-sup constants in the subsequent sections.

\subsubsection{Temporal Operators}

First, let us form temporal matrices required for the evaluation of the PetrovGalerkin finite element semilinear form. We will explicitly determine the entries of the matrices (i.e. the inner products) for our particular choice of basis functions, which are later required to construct a time-marching interpretation. The PetrovGalerkin temporal matrices $\mathbf{M}_{h}^{\text {time }} \in \mathbb{R}^{K \times K}$ and $\dot{\mathbf{M}}_{h}^{\text {time }} \in \mathbb{R}^{K \times K}$ are given by

$$
\begin{aligned}
& \left(\dot{\mathbf{M}}_{\Delta t}^{\mathrm{time}}\right)_{l k}=\left(\dot{\sigma}^{k}, \tau^{l}\right)_{L^{2}(I)}=\delta_{k, l}-\delta_{k+1, l} \\
& \left(\mathbf{M}_{\Delta t}^{\mathrm{time}}\right)_{l k}=\left(\sigma^{k}, \tau^{l}\right)_{L^{2}(I)}=\frac{\Delta t^{l}}{2}\left(\delta_{k, l}+\delta_{k+1, l}\right),
\end{aligned}
$$

where $\delta_{k, l}$ is the Kronecker delta, and $\Delta t^{l} \equiv\left|I^{l}\right|=t^{l}-t^{l-1}$. Note that, with our particular choice of basis functions for $S_{\Delta t}$ and $Q_{\Delta t}$, the matrices are lower bidiagonal. The triple product resulting from the trilinear form evaluates to

$$
\left(\sigma^{k} \sigma^{m}, \tau^{l}\right)_{L^{2}(I)}=\frac{\Delta t^{l}}{6}\left(2 \delta_{k, l} \delta_{m, l}+\delta_{k, l} \delta_{m+1, l}+\delta_{k+1, l} \delta_{m, l}+2 \delta_{k+1, l} \delta_{m+1, l}\right)
$$

(no sum implied on $l$ ). 
In addition, evaluation of the $\mathcal{X}_{\delta}$ inner product requires matrices $\dot{\mathbf{M}}_{\Delta t}^{S} \in \mathbb{R}^{K \times K}$ and $\overline{\mathbf{M}}_{\Delta t}^{S} \in \mathbb{R}^{K \times K}$ associated with $S_{\Delta t}$ given by

$$
\begin{aligned}
& \left(\dot{\mathbf{M}}_{\Delta t}^{S}\right)_{l k}=\left(\dot{\sigma}^{k}, \dot{\sigma}^{l}\right)_{L^{2}(I)}=-\frac{1}{\Delta t^{l}} \delta_{k+1, l}+\left(\frac{1}{\Delta t^{l}}+\frac{1}{\Delta t^{l+1}}\right) \delta_{k, l}-\frac{1}{\Delta t^{l+1}} \delta_{k-1, l} \\
& \left(\overline{\mathbf{M}}_{\Delta t}^{S}\right)_{l k}=\left(\bar{\sigma}^{k}, \bar{\sigma}^{l}\right)_{L^{2}(I)}=\frac{\Delta t^{l}}{4} \delta_{k+1, l}+\frac{\Delta t^{l}+\Delta t^{l+1}}{4} \delta_{k, l}+\frac{\Delta t^{l+1}}{4} \delta_{k-1, l} .
\end{aligned}
$$

Because the support of the basis functions are unaltered by differentiation or the averaging operation, both $\dot{\mathbf{M}}_{\Delta t}^{S}$ and $\overline{\mathbf{M}}_{\Delta t}^{S}$ are tridiagonal. Finally, the evaluation of the $\mathcal{Y}$ inner product requires a matrix $\mathbf{M}_{\Delta t}^{Q} \in \mathbb{R}^{K \times K}$ associated with $Q_{\Delta t}$ given by

$$
\left(\mathbf{M}_{\Delta t}^{Q}\right)_{l k}=\left(\tau^{k}, \tau^{l}\right)_{L^{2}(I)}=\Delta t^{l} \delta_{k, l}
$$

Because $\tau^{k}, k=1, \ldots, K$, have element-wise compact support, $\mathbf{M}_{\Delta t}^{Q}$ is a diagonal matrix.

\subsubsection{Spatial Operators}

The spatial matrices $\mathbf{M}_{h}^{\text {space }} \in \mathbb{R}^{\mathcal{N} \times \mathcal{N}}$ and $\mathbf{A}_{h}^{\text {space }} \in \mathbb{R}^{\mathcal{N} \times \mathcal{N}}$ associated with the $L^{2}(\Omega)$ inner product and the bilinear form $a(\cdot, \cdot)$ are given by

$$
\left(\mathbf{M}_{h}^{\text {space }}\right)_{j i}=\left(\phi_{i}, \phi_{j}\right)_{H} \quad \text { and } \quad\left(\mathbf{A}_{h}^{\text {space }}\right)_{j i}=a\left(\phi_{i}, \phi_{j}\right)
$$

To simplify the notation, let us denote the spatial basis coefficients for time $t^{k}$ by vector $\boldsymbol{w}^{k} \in \mathbb{R}^{\mathcal{N}}$, i.e. the $j$-th entry of $\boldsymbol{w}^{k}$ is $\left(\boldsymbol{w}^{k}\right)_{j}=w_{j}^{k}$. The vector $\boldsymbol{z}^{m} \in \mathbb{R}^{\mathcal{N}}$ is defined similarly. Then, we can express the action of the quadratic term in terms of a function $\boldsymbol{b}_{h}^{\text {space }}: \mathbb{R}^{\mathcal{N}} \times \mathbb{R}^{\mathcal{N}} \rightarrow \mathbb{R}^{\mathcal{N}}$, the $j$-th component of the whose output is given by

$$
\left(\boldsymbol{b}_{h}^{\text {space }}\left(\boldsymbol{w}^{k}, \boldsymbol{z}^{m}\right)\right)_{j}=\sum_{i, n=1}^{\mathcal{N}} w_{i}^{k} z_{n}^{m} b\left(\phi_{i}, \phi_{n}, \phi_{j}\right) .
$$

\subsubsection{Space-Time Operators: Burgers' Equation}

Combining the expressions for the temporal inner products and the spatial operators, the space-time forms evaluated against the test function $\tau^{l} \otimes \phi_{j}$ may be 
expressed as

$$
\begin{aligned}
\mathcal{M}\left(w_{\delta}, \tau^{l} \otimes \phi_{j}\right) & =\sum_{k=1}^{K} \sum_{i=1}^{\mathcal{N}} w_{i}^{k}\left(\dot{\sigma}^{k}, \tau^{l}\right)_{L^{2}(I)}\left(\phi_{i}, \phi_{j}\right)_{H}=\left(\mathbf{M}_{h}^{\text {space }}\left(\boldsymbol{w}^{l}-\boldsymbol{w}^{l-1}\right)\right)_{j} \\
\mathcal{A}\left(w_{\delta}, \tau^{l} \otimes \phi_{j}\right) & =\sum_{k=1}^{K} \sum_{i=1}^{\mathcal{N}} w_{i}^{k}\left(\sigma^{k}, \tau^{l}\right)_{L^{2}(I)} a\left(\phi_{i}, \phi_{j}\right)=\frac{\Delta t^{l}}{2}\left(\mathbf{A}_{h}^{\text {space }}\left(\boldsymbol{w}^{l}+\boldsymbol{w}^{l-1}\right)\right)_{j} \\
\mathcal{B}\left(w_{\delta}, z_{\delta}, \tau^{l} \otimes \phi_{j}\right) & =\sum_{k, m=1}^{K} \sum_{i, n=1}^{\mathcal{N}} w_{i}^{k} z_{n}^{m}\left(\sigma^{k} \sigma^{m}, \tau^{l}\right)_{L^{2}(I)} b\left(\phi_{i}, \phi_{n}, \phi_{j}\right) \\
& =\sum_{i, n=1}^{\mathcal{N}} \frac{\Delta t^{l}}{6}\left(2 w_{i}^{l} z_{n}^{l} b\left(\phi_{i}, \phi_{n}, \phi_{j}\right)+w_{i}^{l} z_{n}^{l-1} b\left(\phi_{i}, \phi_{n}, \phi_{j}\right)\right. \\
& =\frac{\Delta t^{l}}{6}\left(2 w_{i}^{l-1} z_{n}^{l} b\left(\phi_{i}, \phi_{n}, \phi_{j}\right)+2 w_{i}^{l-1} z_{n}^{l-1} b\left(\phi_{i}, \phi_{n}, \phi_{j}\right)\right) \\
& \left.\left.+\boldsymbol{w}_{h}^{l}, \boldsymbol{z}^{l}\right)+\boldsymbol{b}_{h}^{\text {space }}\left(\boldsymbol{w}^{l-1}, \boldsymbol{z}^{l}\right)+2 \boldsymbol{w}_{h}^{l}, \boldsymbol{z}^{l-1}\right)
\end{aligned}
$$

The trilinear form further simplifies when the first two arguments are the same, as in the case for the semilinear form of the Burgers' equation, Eq. (2.4); i.e.

$\mathcal{B}\left(w_{\delta}, w_{\delta}, \tau^{l} \otimes \phi_{j}\right)=\frac{\Delta t^{l}}{3}\left(\boldsymbol{b}_{h}^{\text {space }}\left(\boldsymbol{w}^{l}, \boldsymbol{w}^{l}\right)+\boldsymbol{b}_{h}^{\text {space }}\left(\boldsymbol{w}^{l}, \boldsymbol{w}^{l-1}\right)+\boldsymbol{b}_{h}^{\text {space }}\left(\boldsymbol{w}^{l-1}, \boldsymbol{w}^{l-1}\right)\right)$.

In addition, the integration of the forcing function using the trapezoidal rule results in

$$
\begin{aligned}
\mathcal{F}\left(\tau^{l} \otimes \phi_{j} ; \mu\right) & \equiv \mu \cdot \int_{I}\left\langle g_{0}(t), \tau^{l} \otimes \phi_{j}\right\rangle_{V^{\prime} \times V} d t \approx \Delta t^{l} \mu \cdot \frac{1}{2}\left\langle g_{0}\left(t^{l}\right)+g_{0}\left(t^{l-1}\right), \phi_{j}\right\rangle_{V^{\prime} \times V} \\
& =\Delta t^{l} \mu \frac{1}{2}\left(\boldsymbol{g}_{0, h}^{l}+\boldsymbol{g}_{0, h}^{l-1}\right)_{j},
\end{aligned}
$$

where $\boldsymbol{g}^{l} \in \mathbb{R}^{\mathcal{N}}$ with $\left(\boldsymbol{g}_{h}^{l}\right)_{j}=\left\langle g\left(t^{l}\right), \phi_{j}\right\rangle_{V^{\prime} \times V}$. Combining the expressions for our particular choice of the Petrov-Galerkin test functions, the finite element residual statement, Eq. (2.5), may be simplified to

$$
\begin{aligned}
& \frac{1}{\Delta t^{l}} \mathbf{M}_{h}^{\text {space }}\left(\boldsymbol{w}^{l}-\boldsymbol{w}^{l-1}\right)+\frac{1}{2} \mathbf{A}_{h}^{\text {space }}\left(\boldsymbol{w}^{l}+\boldsymbol{w}^{l-1}\right) \\
& \quad+\frac{1}{3}\left(\boldsymbol{b}_{h}^{\text {space }}\left(\boldsymbol{w}^{l}, \boldsymbol{w}^{l}\right)+\boldsymbol{b}_{h}^{\text {space }}\left(\boldsymbol{w}^{l}, \boldsymbol{w}^{l-1}\right)+\boldsymbol{b}_{h}^{\text {space }}\left(\boldsymbol{w}^{l-1}, \boldsymbol{w}^{l-1}\right)\right)-\mu \frac{1}{2}\left(\boldsymbol{g}_{0, h}^{l}+\boldsymbol{g}_{0, h}^{l-1}\right),
\end{aligned}
$$

for $l=1, \ldots, K$, with $\boldsymbol{w}^{0}=0$. Note that the treatment of the linear terms are identical to that resulting from the Crank-Nicolson time stepping, whereas the quadratic term results in a different form. In any event, the Petrov-Galerkin space-time formulation admits a time-marching interpretation; the solution can be obtained by sequentially solving $K$ systems of nonlinear equations, each having $\mathbb{R}^{\mathcal{N}}$ unknowns; thus, the computational cost is equivalent to that of the Crank-Nicolson scheme. 


\subsubsection{Space-Time Operators: $\mathcal{X}_{\delta}$ and $\mathcal{Y}_{\delta}$ Inner Products}

Combining the temporal matrices with the spatial matrices introduced in Section 2.3 , we can express the matrix associated with the $\mathcal{X}_{\delta}$ inner product, $\mathbf{X} \in$ $\mathbb{R}^{(K \cdot \mathcal{N}) \times(K \cdot \mathcal{N})}$, as

$$
\mathbf{X}=\dot{\mathbf{M}}_{\Delta t}^{S} \otimes\left(\mathbf{M}_{h}^{\text {space }}\left(\mathbf{A}_{h}^{\text {space }}\right)^{-1} \mathbf{M}_{h}^{\text {space }}\right)+\overline{\mathbf{M}}_{\Delta t}^{S} \otimes \mathbf{A}_{h}^{\text {space }} .
$$

Note that $\mathbf{X}$ is block-tridiagonal. Similarly, the matrix associated with the $\mathcal{Y}_{\delta}$ inner product, $\mathbf{Y} \in \mathbb{R}^{(K \cdot \mathcal{N}) \times(K \cdot \mathcal{N})}$, is given by

$$
\mathbf{Y}=\mathbf{M}_{\Delta t}^{Q} \otimes \mathbf{A}_{h}^{\text {space }} .
$$

The matrix $\mathbf{Y}$ is block diagonal because $\mathbf{M}_{\Delta t}^{Q}$ is diagonal. Note that the norm induced by the $\overline{\mathbf{M}}_{\Delta t}^{S} \otimes \mathbf{A}_{h}^{\text {space }}$ part of the $\mathbf{X}$ matrix is identical to the usual norm for the Crank-Nicolson scheme, i.e.

$$
\begin{aligned}
\left\{w_{i}^{k}\right\}^{T}\left(\overline{\mathbf{M}}_{\Delta t}^{S} \otimes \mathbf{A}_{h}^{\text {space }}\right)\left\{w_{i}^{k}\right\} & =\left\|w_{\delta}\right\|_{\mathrm{CN}}^{2} \\
& \equiv \sum_{k=1}^{K}\left(\frac{1}{2}\left(\boldsymbol{w}^{k}+\boldsymbol{w}^{k-1}\right)\right)^{T} \mathbf{A}_{h}^{\text {space }}\left(\frac{1}{2}\left(\boldsymbol{w}^{k}+\boldsymbol{w}^{k-1}\right)\right),
\end{aligned}
$$

where $\left\{w_{i}^{k}\right\} \in \mathbb{R}^{K \cdot \mathcal{N}}$ is a vector of space-time basis coefficients for $w_{\delta}$. The identity - together with the equivalence of our space-time Petrov-Galerkin formulation with the Crank-Nicolson scheme for linear problems - suggests that the inclusion of the averaging operator in our $\mathcal{X}_{\delta}$ norm is rather natural for the particular scheme we consider.

\section{Certified Space-Time Reduced-Basis Approximation}

\section{1. $N_{\mu}-p$ Interpolation-Based Approximation}

Here, we introduce a simple reduced-basis approximation procedure based on solution interpolation (rather than projection). We choose interpolation as it is less expensive than projection, sufficiently accurate in one parameter dimension, and also facilitates construction of an inf-sup lower bound as we will show in Section 3.2.2.

We consider an $h p$-decomposition (or, more specifically, $N_{\mu}-p$ decomposition) of the parameter domain $\mathcal{D}$ as considered in Eftang et al. ${ }^{3}$ In particular, we partition $\mathcal{D} \subset \mathbb{R}^{1}$ into $N_{\mu}$ subdomains, $\mathcal{D}_{j}=\left[\mu_{j}^{L}, \mu_{j}^{U}\right], j=1, \ldots, N_{\mu}$, and approximate the solution variation over each subdomain using a degree- $p$ polynomial. On each $\mathcal{D}_{j}$, we use $p+1$ Chebyshev-Lobatto nodes

$$
\mu_{j, k}=\frac{\mu-\mu^{L}}{\mu^{U}-\mu^{L}}\left[\frac{1}{2} \cos \left(\frac{2 k-1}{2(p+1)} \pi\right)+\frac{1}{2}\right], \quad k=1, \ldots, p+1,
$$

as the interpolation points. At each interpolation point, we obtain the truth solution $u_{j, k} \equiv u_{\delta}\left(\mu_{j, k}\right)$ by solving the finite element approximation, Eq. (2.5). (For notational simplicity, we will suppress the subscript $\delta$ for the finite element truth 
solutions from hereon.) Then, we construct our reduced basis approximation to $u=u(\mu)$ by a direct sum of $N_{\mu}$ polynomials

$$
\tilde{u}^{p}=\bigoplus_{j=1}^{N_{\mu}} \tilde{u}_{j}^{p},
$$

where $\tilde{u}_{j}^{p}$ is a degree- $p$ polynomial over $\mu \in \mathcal{D}_{j}$ given by

$$
\tilde{u}_{j}^{p}(\mu)=\sum_{k=1}^{p+1} u_{j, k} \psi_{k}^{p}(\mu)
$$

for $j=1, \ldots, N_{\mu}$. Here $\psi_{k}^{p}$ is the degree- $p$ Chebyshev polynomial corresponding to the $k$-th interpolation point, i.e. $\psi_{k}^{p} \in \mathbb{P}^{p}\left(\mathcal{D}_{j}\right)$ such that $\psi_{k}^{p}\left(x_{l}\right)=\delta_{k, l}$, $k, l=1, \ldots, p+1$. Note that, unlike in the classical time-marching formulation, ${ }^{5,9,7}$ the computational cost of constructing the reduced-basis approximation using our space-time formulation is independent of the number of time steps, $K$. In this work, we do not assess the relative approximation properties of classical time-marching formulation (e.g. POD-Greedy) and our $N_{\mu}-p$ interpolation method.

\subsection{Brezzi-Rappaz-Raviart Theory}

Our a posteriori error estimate for the Burgers' equation is a straightforward application of the Brezzi-Rappaz-Raviart (BRR) theory ${ }^{1}$. The following proposition states the main results of the theory; detailed proof for a general case is provided in the original paper ${ }^{1}$ and for quadratic nonlinearity is presented by Veroy and Patera. ${ }^{14}$

Proposition 3.1. Let the dual norm of the residual, the inf-sup constant, and the $L^{4}-\mathcal{X}_{\delta}$ Sobolev embedding constant be given by

$$
\begin{aligned}
\epsilon^{p}(\mu) & \equiv \sup _{v \in \mathcal{Y}} \frac{\mathcal{G}\left(\tilde{u}^{p}(\mu), v ; \mu\right)}{\|v\|_{\mathcal{Y}}}, \\
\beta^{p}(\mu) & \equiv \inf _{w \in \mathcal{X}_{\delta}} \sup _{v \in \mathcal{Y}} \frac{\partial \mathcal{G}\left(w, \tilde{u}^{p}(\mu), v\right)}{\|w\|_{\mathcal{X}_{\delta}}\|v\|_{\mathcal{Y}}}, \\
\rho & \equiv \sup _{w \in \mathcal{X}_{\delta}} \frac{\|w\|_{L^{4}\left(I ; L^{4}(\Omega)\right)}}{\|w\|_{\mathcal{X}_{\delta}}} .
\end{aligned}
$$

In addition, let $\beta_{L B}^{p}(\mu)$ be a lower bound of $\beta^{p}(\mu)$, i.e. $\beta_{L B}^{p}(\mu) \leq \beta^{p}(\mu), \forall \mu \in \mathcal{D}$. Let the proximately indicator be $\tau^{p}(\mu) \equiv 2 \rho^{2} \epsilon^{p}(\mu) /\left(\beta_{L B}^{p}\right)^{2}(\mu)$. Then, for $\tau^{p}(\mu)<1$, there exists a unique solution $u(\mu) \in \mathfrak{B}\left(\tilde{u}^{p}(\mu), \beta^{p}(\mu) / \rho^{2}\right)$, where $\mathfrak{B}(z, r) \equiv\{x \in$ $\left.\mathcal{X}_{\delta}:\|x-z\|_{\mathcal{X}_{\delta}}<r\right\}$. Furthermore, $\left\|u(\mu)-\tilde{u}^{p}(\mu)\right\|_{\mathcal{X}_{\delta}} \leq \Delta^{p}(\mu)$ where

$$
\Delta^{p}(\mu) \equiv \frac{\beta_{L B}^{p}(\mu)}{\rho^{2}}\left(1-\sqrt{1-\tau^{p}(\mu)}\right) .
$$

Proof. Proof is provided in, for example, in Veroy and Patera. ${ }^{14}$ 
The following subsections detail the computation of the three key ingredients of the BRR theory: the dual norm of residual $\epsilon_{p}(\mu)$; the inf-sup constant, $\beta_{\mathrm{LB}, p}(\mu)$; and the $L^{4}-\mathcal{X}_{\delta}$ Sobolev embedding constant $\rho$. In particular, we will present efficient means of computing these variables that permits offline-online decomposition.

\subsubsection{Residual Evaluation}

Here, we briefly review a technique for efficiently computing the dual norm of the residual in the online stage, the technique originally presented by Veroy et al. ${ }^{14} \mathrm{We}$ first note that $\epsilon^{p}(\mu) \equiv\left\|\mathcal{G}\left(\tilde{u}^{p}(\mu), \cdot ; \mu\right)\right\|_{\mathcal{Y}^{\prime}}=\left\|\hat{e}^{p}\right\|_{\mathcal{Y}}$, where the Riesz representor of the residual is given by $\hat{e}^{p} \equiv R \mathcal{G}\left(\tilde{u}^{p}(\mu), \cdot ; \mu\right) \in \mathcal{Y}$ and satisfies

$$
\begin{aligned}
\left(\hat{e}^{p}, v\right)_{\mathcal{Y}}= & \mathcal{G}\left(\tilde{u}^{p}(\mu), v ; \mu\right) \\
= & \sum_{k=1}^{p+1} \psi_{k}^{p}(\mu)\left[\mathcal{M}\left(\dot{u}_{k}, v\right)+\mathcal{A}\left(u_{k}, v\right)\right] \\
& +\sum_{k, l=1}^{p+1} \psi_{k}^{p}(\mu) \psi_{l}^{p}(\mu) \mathcal{B}\left(u_{k}, u_{l}, v\right)-\mu \cdot \mathcal{F}_{0}(v), \quad \forall v \in \mathcal{Y} .
\end{aligned}
$$

Let us introduce (pieces of) Riesz representators $\chi^{0},\left\{\chi_{k}^{1}\right\}_{k=1}^{p+1}$, and $\left\{\chi_{k l}^{2}\right\}_{k, l=1}^{p+1}$ of the residual contribution from the linear, bilinear, and trilinear form, respectively, for the snapshots according to

$$
\begin{aligned}
\left(\chi^{0}, v\right)_{\mathcal{Y}} & =\mathcal{F}_{0}(v), \quad \forall v \in \mathcal{Y}, \\
\left(\chi_{k}^{1}, v\right)_{\mathcal{Y}} & =\mathcal{M}\left(\hat{u}_{k}, v\right)+\mathcal{A}\left(u_{k}, v\right), \quad \forall v \in \mathcal{Y}, k=1, \ldots, p+1, \\
\left(\chi_{k l}^{2}, v\right)_{\mathcal{Y}} & =\mathcal{B}\left(u_{k}, u_{l}, v\right), \quad \forall v \in \mathcal{Y}, k, l=1, \ldots, p+1 .
\end{aligned}
$$

Then, we can express $\hat{e}^{p}$ as

$$
\hat{e}^{p}=\mu \cdot \chi^{0}+\sum_{k=1}^{p+1} \psi_{k}^{p}(\mu) \chi_{k}^{1}+\sum_{k, l=1}^{p+1} \psi_{k}^{p}(\mu) \psi_{l}^{p}(\mu) \chi_{k l}^{2} .
$$

The dual norm of the residual can be expressed as

$$
\begin{aligned}
\left\|\hat{e}^{p}\right\|_{\mathcal{Y}}= & \mu^{2}\left(\chi^{0}, \chi^{0}\right) \mathcal{Y}+2 \mu \sum_{m=1}^{p+1}\left(\chi^{0}, \chi_{m}^{1}\right) \mathcal{Y}+2 \mu \sum_{m, n=1}^{p+1}\left(\chi^{0}, \chi_{m n}^{2}\right) \mathcal{Y} \\
& +\sum_{k, m=1}^{p+1} \psi_{k}^{p}(\mu) \psi_{m}^{p}(\mu)\left(\chi_{k}^{1}, \chi_{m}^{1}\right) \mathcal{Y}+2 \sum_{k, m, n=1}^{p+1} \psi_{k}^{p}(\mu) \psi_{m}^{p}(\mu) \psi_{n}^{p}(\mu)\left(\chi_{k}^{1}, \chi_{m n}^{2}\right) \mathcal{Y} \\
& +\sum_{k, l, m, n=1}^{p+1} \psi_{k}^{p}(\mu) \psi_{l}^{p}(\mu) \psi_{m}^{p}(\mu) \psi_{n}^{p}(\mu)\left(\chi_{k l}^{2}, \chi_{m n}^{2}\right) \mathcal{Y}
\end{aligned}
$$

The offline-online decomposition is clear from the expression. In the offline stage, we first solve Eq. (3.1)-(3.3) to obtain the Riesz representors $\chi^{0},\left\{\chi_{k}^{1}\right\}_{k=1}^{p+1}$, and $\left\{\chi_{k l}^{2}\right\}_{k, l=1}^{p+1}$. Note that there are $1+(p+1)+(p+1)^{2}$ representors, each requiring 
a $\mathcal{Y}$-solve. Recalling that the matrix associated with the $\mathcal{Y}$ inner product is given by $\mathbf{Y}=\mathbf{M}_{\Delta t}^{Q} \otimes \mathbf{A}_{h}^{\text {space }}$, each $\mathcal{Y}$-solve requires $K$ inversions of the $\mathbf{A}_{h}^{\text {space }}$ operator, where $K$ is the number of time steps. It is important to note that the computation of the representators does not require a solution of a coupled space-time system, as the matrix $\mathbf{Y}$ is block diagonal. In other words, the computational cost is not higher than that for the classical time-marching reduced basis formulation. After computing the representators, we compute the $\mathcal{Y}$ inner product of all permutation of representators, i.e. $\left(\chi^{0}, \chi^{0}\right) \mathcal{Y},\left(\chi^{0}, \chi_{k}^{1}\right) \mathcal{y}$, etc.

In the online stage, we obtain the dual norm of the residual by evaluating Eq. (3.4) using the inner products computed in the offline stage. The computational cost scales as $(p+1)^{4}$ and is independent of the cost of truth discretization. Note that, unlike in the classical reduced-basis formulation based on time-marching, the online residual evaluation cost of our space-time formulation is independent of the number of time steps, $K$.

\subsubsection{Inf-Sup Constant and its Lower Bound}

Here, we present a procedure for computing an inf-sup lower bound, $\beta_{\mathrm{LB}}^{p}(\mu)$, that permits offline-online decomposition. The particular procedure presented is specifically designed for the $N_{\mu}-p$ interpolation-based reduced basis approximation introduced in Section 3.1. Let us first define the supremizing operator $S_{j}^{c}: \mathcal{X}_{\delta} \rightarrow \mathcal{Y}_{\delta}$ associated with the solution $u_{j}^{c}=u\left(\mu_{j}^{c}\right)$ at the centroid of $\mathcal{D}_{j}, \mu_{j}^{c}$, by

$$
\left(S_{j}^{c} w, v\right)_{\mathcal{Y}}=\partial \mathcal{G}\left(w, u_{j}^{c}, v\right), \quad \forall w \in \mathcal{X}_{\delta}, \forall v \in \mathcal{Y}_{\delta},
$$

for $j=1, \ldots, N_{\mu}$. The inf-sup constant about $u_{j}^{c}$ is given by

$$
\beta_{j}^{c}=\inf _{w \in \mathcal{X}_{\delta}} \frac{\left\|S_{j}^{c} w\right\|_{\mathcal{Y}}}{\|w\|_{\mathcal{X}_{\delta}}} .
$$

Let us also introduce the following correction factors at interpolation points,

$$
\beta_{j, k}^{-} \equiv \inf _{w \in \mathcal{X}} \frac{\partial \mathcal{G}\left(w, u_{j, k}, S_{j}^{c} w\right)}{\left\|S_{j}^{c} w\right\|_{\mathcal{Y}}^{2}} \quad \text { and } \quad \beta_{j, k}^{+} \equiv \sup _{w \in \mathcal{X}} \frac{\partial \mathcal{G}\left(w, u_{j, k}, S_{j}^{c} w\right)}{\left\|S_{j}^{c} w\right\|_{\mathcal{Y}}^{2}}
$$

for $k=1, \ldots, p+1$ and $j=1, \ldots, N_{\mu}$. Then, we construct an inf-sup lower bound according to

$$
\beta_{\mathrm{LB}, j}^{p}(\mu)=\beta_{j}^{c} \cdot\left(\sum_{\substack{k=1, \ldots, p+1 \\ \psi_{k}^{p}(\mu)>0}} \beta_{j, k}^{-} \psi_{k}^{p}(\mu)+\sum_{\substack{k=1, \ldots, p+1 \\ \psi_{k}^{p}(\mu)<0}} \beta_{j, k}^{+} \psi_{k}^{p}(\mu)\right), \quad \forall \mu \in \mathcal{D}_{j} .
$$

Let us denote the lower bound over the entire $\mathcal{D}$ by $\beta_{\mathrm{LB}}^{p}$, which is simply the direct sum of the piecewise lower bounds, i.e. $\beta_{\mathrm{LB}}^{p}=\oplus_{j=1}^{N_{\mu}} \beta_{\mathrm{LB}, j}^{p}$. We have the following proposition:

Proposition 3.2. The inf-sup lower bound constructed using the above procedure satisfies $\beta_{L B}^{p}(\mu) \leq \beta^{p}(\mu), \forall \mu \in \mathcal{D}$. 
Proof. Let us show that the $\beta_{\mathrm{LB}, j}^{p}(\mu) \leq \beta^{p}(\mu)$ for $\mu \in \mathcal{D}_{j}$ for each $j=1, \ldots, N_{\mu}$. Since $S_{j}^{c} w \in \mathcal{Y}, \forall w \in \mathcal{X}_{\delta}$, we can bound the inf-sup constant from below as

$$
\begin{aligned}
\beta^{p}(\mu) & \equiv \inf _{w \in \mathcal{X}_{\delta}} \sup _{v \in \mathcal{Y}} \frac{\partial \mathcal{G}\left(w, \tilde{u}^{p}(\mu), v\right)}{\|w\|_{\mathcal{X}_{\delta}}\|v\|_{\mathcal{Y}}}=\inf _{w \in \mathcal{X}_{\delta}} \sup _{v \in \mathcal{Y}} \sum_{k=1}^{p+1} \psi_{k}^{p}(\mu) \frac{\partial \mathcal{G}\left(w, u_{j, k}, v\right)}{\|w\|_{\mathcal{X}_{\delta}}\|v\|_{\mathcal{Y}}} \\
& \geq \inf _{w \in \mathcal{X}_{\delta}} \sum_{k=1}^{p+1} \psi_{k}^{p}(\mu) \frac{\partial \mathcal{G}\left(w, u_{j, k}, S_{j}^{c} w\right)}{\|w\|_{\mathcal{X}_{\delta}}\left\|S_{j}^{c} w\right\|_{\mathcal{Y}}}=\inf _{w \in \mathcal{X}_{\delta}} \sum_{k=1}^{p+1} \psi_{k}^{p}(\mu) \frac{\left\|S_{j}^{c} w\right\|_{\mathcal{Y}}}{\|w\|_{\mathcal{X}_{\delta}}} \frac{\partial \mathcal{G}\left(w, u_{j, k}, S_{j}^{c} w\right)}{\left\|S_{j}^{c} w\right\|_{\mathcal{Y}}^{2}} \\
& =\inf _{w \in \mathcal{X}_{\delta}} \frac{\left\|S_{j}^{c} w\right\|_{\mathcal{Y}}}{\|w\|_{\mathcal{X}_{\delta}}} \sum_{k=1}^{p+1} \psi_{k}^{p}(\mu) \frac{\partial \mathcal{G}\left(w, u_{j, k}, S_{j}^{c} w\right)}{\left\|S_{j}^{c} w\right\|_{\mathcal{Y}}^{2}}, \quad \forall \mu \in \mathcal{D}_{j}, j=1, \ldots, N_{\mu} .
\end{aligned}
$$

Note that we have

$$
\frac{\left\|S_{j}^{c} w\right\|_{\mathcal{Y}}}{\|w\|_{\mathcal{X}_{\delta}}} \geq \inf _{z \in \mathcal{X}_{\delta}} \frac{\left\|S_{j}^{c} z\right\|_{\mathcal{Y}}}{\|z\|_{\mathcal{X}_{\delta}}}=\beta\left(\mu_{j}^{c}\right) \equiv \beta_{j}^{c}>0, \quad \forall w \in \mathcal{X}_{\delta}
$$

and the first term of Eq. (3.7) is bounded below by $\beta_{j}^{c}>0$. The second term involving summation over $p+1$ terms may be may be bounded below by the correction factors defined in Eq. (3.5). Namely, if $\psi_{k}^{p}(\mu)>0$, then we may bound the contribution from the $k$-th term from below by using $\beta_{j, k}^{-}$; if $\psi_{k}^{p}(\mu)<0$, then the contribution may be bounded from below by using $\beta_{j, k}^{+}$. In other words, the final expression of Eq. (3.7) is bounded from below by

$$
\begin{gathered}
\beta^{p}(\mu) \geq\left(\inf _{w \in \mathcal{X}_{\delta}} \frac{\left\|S_{j}^{c} w\right\|_{\mathcal{Y}}}{\|w\|_{\mathcal{X}_{\delta}}}\right)\left(\sum_{\substack{k=1, \ldots, p+1 \\
\psi_{k}^{p}(\mu)>0}} \psi_{k}^{p}(\mu) \inf _{w \in \mathcal{X}_{\delta}} \frac{\partial \mathcal{G}\left(w, u_{j, k}, S_{j}^{c} w\right)}{\left\|S_{j}^{c} w\right\|_{\mathcal{Y}}^{2}}\right. \\
\left.+\sum_{\substack{k=1, \ldots, p+1 \\
\psi_{k}^{p}(\mu)<0}} \psi_{k}^{p}(\mu) \sup _{w \in \mathcal{X}_{\delta}} \frac{\partial \mathcal{G}\left(w, u_{j, k}, S_{j}^{c} w\right)}{\left\|S_{j}^{c} w\right\|_{\mathcal{Y}}^{2}}\right) \\
=\beta_{j}^{c}\left(\sum_{\substack{k=1, \ldots, p+1 \\
\psi_{k}^{p}(\mu)>0}} \beta_{j, k}^{-} \psi_{k}^{p}(\mu)+\sum_{\substack{k=1, \ldots, p+1 \\
\psi_{k}^{p}(\mu)<0}} \beta_{j, k}^{+} \psi_{k}^{p}(\mu)\right), \quad \forall \mu \in \mathcal{D}_{j}, j=1, \ldots, N_{\mu},
\end{gathered}
$$

which concludes the proof.

Remark 3.1. For small intervals, the correction factors are close to unity. To see 
this, we note that

$$
\begin{aligned}
\frac{\left|\partial \mathcal{G}\left(w, u_{j, k}, S_{j}^{c} w\right)\right|}{\left\|S_{j}^{c} w\right\|_{\mathcal{Y}}} & =\frac{\left|\partial \mathcal{G}\left(w, u_{j}^{c}+\left(u_{j, k}-u_{j}^{c}\right), S_{j}^{c} w\right)\right|}{\left\|S_{j}^{c} w\right\|_{\mathcal{Y}}} \\
& \leq \frac{\left|\partial \mathcal{G}\left(w, u_{j}^{c}, S_{j}^{c} w\right)\right|}{\left\|S_{j}^{c} w\right\|_{\mathcal{Y}}}+\frac{\left|\mathcal{B}\left(w, u_{j, k}-u_{j}^{c}, S_{j}^{c} w\right)\right|}{\left\|S_{j}^{c} w\right\|_{\mathcal{Y}}} \\
& \leq 1+\frac{1}{2} \frac{\rho^{2}\|w\|_{\mathcal{X}_{\delta}}\left\|u_{j, k}-u_{j}^{c}\right\|_{\mathcal{X}_{\delta}}\left\|S_{j}^{c} w\right\|_{\mathcal{Y}}}{\beta^{2}\left(\mu_{j}^{c}\right)\|w\|_{\mathcal{X}_{\delta}}^{2}} \\
& \leq 1+\frac{\rho^{2}}{2 \beta^{2}\left(\mu_{j}^{c}\right)}\left(\gamma^{\text {heat }}+\frac{1}{2} \rho^{2}\left\|u_{j}^{c}\right\|_{\mathcal{X}_{\delta}}\right)\left\|u_{j, k}-u_{j}^{c}\right\|_{\mathcal{X}_{\delta}},
\end{aligned}
$$

where $\gamma^{\text {heat }}$ is the continuity constant for the heat equation, i.e.

$$
\gamma^{\text {heat }} \equiv \sup _{w \in \mathcal{X}_{\delta}} \sup _{v \in \mathcal{Y}} \frac{|\mathcal{M}(\dot{w}, v)+\mathcal{A}(w, v)|}{\|w\|_{\mathcal{X}_{\delta}}\|v\|_{\mathcal{Y}}} .
$$

Thus, as $\left|\mathcal{D}_{j}\right| \rightarrow 0$ and $\left\|u_{j, k}-u_{j}^{c}\right\|_{\mathcal{X}_{\delta}} \rightarrow 0$, the correction factors converge to 1 .

Remark 3.2. The inf-sup lower bound construction procedure presented here produces a tighter lower bound than the natural norm Successive Constraint Method $(\mathrm{SCM})^{6}$ that uses the $p+1$ interpolations as the SCM sampling points, i.e.

$$
\beta(\mu) \geq \beta_{\mathrm{LB}, j}^{p}(\mu) \geq \beta_{\mathrm{LB}, \mathrm{SCM}, j}^{p}(\mu), \quad \forall \mu \in \mathcal{D}_{j},
$$

where $\beta_{\mathrm{LB}, \mathrm{SCM}, j}^{p}(\mu)$ is the SCM inf-sup lower bound. A detailed derivation is provided in Appendix B.

Again, the offline-online decomposition is clear from the structure of Eq. (3.6). In the offline stage, for each $\mathcal{D}_{j}$, we evaluate the inf-sup constant at the centroid, $\beta_{j}^{c}$, and correction factors $\beta_{j, k}^{ \pm}$at each of the $p+1$ interpolation points. The online stage consists of identifying the parameter subdomain $\mathcal{D}_{j}$ to which $\mu$ belongs and evaluating $\beta_{\mathrm{LB}, j}^{p}(\mu)$ using Eq. (3.6).

Let us demonstrate that none of the offline computations require solutions to a fully-coupled space-time problem, and the computational cost scales linearly with $K$. The inf-sup constant at the centroid, $\beta_{j}^{c}$, can be obtained by finding the largest eigenvalue of a generalized eigenproblem $\mathbf{P v}=\lambda \mathbf{Q v}$ with

$$
\begin{aligned}
& \mathbf{P}=\mathbf{X}=\dot{\mathbf{M}}_{\Delta t}^{S} \otimes\left(\mathbf{M}_{h}^{\text {space }}\left(\mathbf{A}_{h}^{\text {space }}\right)^{-1} \mathbf{M}_{h}^{\text {space }}\right)+\overline{\mathbf{M}}_{\Delta t}^{S} \otimes \mathbf{A}_{h}^{\text {space }} \\
& \mathbf{Q}=\left(\mathbf{G}^{c}\right)^{T} \mathbf{Y}^{-1} \mathbf{G}^{c}
\end{aligned}
$$

and setting $\beta_{j}^{c}=\lambda_{\max }^{-1 / 2}$. Here, $\mathbf{G}^{c} \in \mathbb{R}^{(K \cdot \mathcal{N}) \times(K \cdot \mathcal{N})}$ is the Jacobian matrix of the residual operator linearized about $u^{c}=u\left(\mu_{j}^{c}\right)$; the $(l i)(k j)$ entry of the matrix is given by

$$
\left(\mathbf{G}^{c}\right)_{(l i)(k j)}=\partial \mathcal{G}\left(\sigma^{k} \otimes \phi_{j}, u^{c}, \tau^{l} \otimes \phi_{i}\right) .
$$

Note that $\mathbf{G}^{c}$ is block lower bidiagonal due to our choice of the basis functions for the spaces $S_{\Delta t}$ and $Q_{\Delta t}$ in the Petrov-Galerkin formulation. If the eigenproblem is 
solved using an Arnoldi-based method, each Arnoldi step requires action of $\mathbf{P}, \mathbf{Q}$ and $\mathbf{Q}^{-1}$ on a vector in $\mathbb{R}^{K \cdot \mathcal{N}}$. The application of $\mathbf{P}$ requires $\mathcal{O}(K)$ operations due to the tensor-product structure of the matrices that constitutes $\mathbf{X}$; for instance, to compute $\overline{\mathbf{M}}_{\Delta t}^{S} \otimes \mathbf{A}_{h}^{\text {space }} \mathbf{v}$, we first compute $\mathbf{A}_{h}^{\text {space }} \mathbf{v}^{k}, k=1, \ldots, K$, and then take a linear combination of (at most) three $\mathbf{A}_{h}^{\text {space }} \mathbf{v}^{k}$ 's according to the weights specified in $\overline{\mathbf{M}}_{\Delta t}^{S}$. The application of $\mathbf{Q}$ requires application of $\mathbf{G}^{c},\left(\mathbf{G}^{c}\right)^{T}$, and $\mathbf{Y}^{-1}$, each of which requires $\mathcal{O}(K)$ operations due to the block bidiagonal or block diagonal structure of the matrices. Finally, the application of $\mathbf{Q}^{-1}=\left(\mathbf{G}^{c}\right)^{-1} \mathbf{Y}\left(\mathbf{G}^{c}\right)^{-T}$ requires: 1) $\left(\mathbf{G}^{c}\right)^{-T}$, which corresponds to a backward solve of a linearized $K$-step time marching problem; 2) $\mathcal{Y}$, which requires application of $\mathbf{A}_{h}^{\text {space }}$ onto $K$ spatial vectors; and 3) $\left(\mathbf{G}^{c}\right)^{-1}$, which corresponds to a forward solve of a linearized $K$ step time marching problem. Thus, each Arnoldi step of the inf-sup eigenproblem requires $\mathcal{O}(K)$ operations.

The calculation of the correction factors require the extreme eigenvalues of a generalized eigenproblem $\mathbf{P v}=\lambda \mathbf{Q v}$ with

$$
\begin{aligned}
& \mathbf{P}=\frac{1}{2}\left(\left(\mathbf{G}^{c}\right)^{T} \mathbf{Y}^{-1} \mathbf{G}^{k}+\left(\mathbf{G}^{k}\right)^{T} \mathbf{Y}^{-1} \mathbf{G}^{c}\right) \\
& \mathbf{Q}=\left(\mathbf{G}^{c}\right)^{T} \mathbf{Y}^{-1} \mathbf{G}^{c} .
\end{aligned}
$$

Here, $\mathbf{G}^{k} \in \mathbb{R}^{(K \cdot \mathcal{N}) \times(K \cdot \mathcal{N})}$ is the Jacobian matrix corresponding to the residual operator linearized about the solution at the interpolation point $u_{j, k}$. Application of $\mathbf{P}$ again requires $\mathcal{O}(K)$ operations due to the block bidiagonal and block diagonal structure of $\mathbf{G}^{c}$ and $\mathbf{Y}$, respectively. The $\mathbf{Q}$ matrix is identical to that used for the inf-sup constant calculation; thus, application of $\mathbf{Q}$ and $\mathbf{Q}^{-1}$ can be carried out in $\mathcal{O}(K)$ operations.

\subsubsection{Sobolev Embedding Constant}

The final piece required for the BRR theory is the $L^{4}-\mathcal{X}_{\delta}$ Sobolev embedding constant. Details of approximating the embedding constant is provided in Appendix A; here we state the main results. Due to the nonlinearity, we have not been able to analyze the $L^{4}-\mathcal{X}_{\delta}$ embedding problem analytically. However, we can analyze closely related linear problems: $L^{2}-\mathcal{X}$ embedding and $L^{2}-\mathcal{X}_{\delta}$ embedding. Using the Fourier decomposition in space and time, we can show that the $L^{2}-\mathcal{X}$ embedding constant is bounded by

$$
\theta \equiv \sup _{w \in \mathcal{X}} \frac{\|w\|_{L^{2}\left(I ; L^{2}(\Omega)\right)}}{\|w\|_{\mathcal{X}}} \leq\left(\frac{1}{4 T^{2}}+\pi^{2}\right)^{-1 / 2}
$$

for $\Omega=(0,1)$ and $I=(0, T]$ with $T>1 /(4 \pi)$. An upper bound for the $L^{2}-\mathcal{X}_{\delta}$ embedding constant can also be analytically found for constant time-stepping cases using the Fourier decomposition in space and von Neumann analysis in time; the 


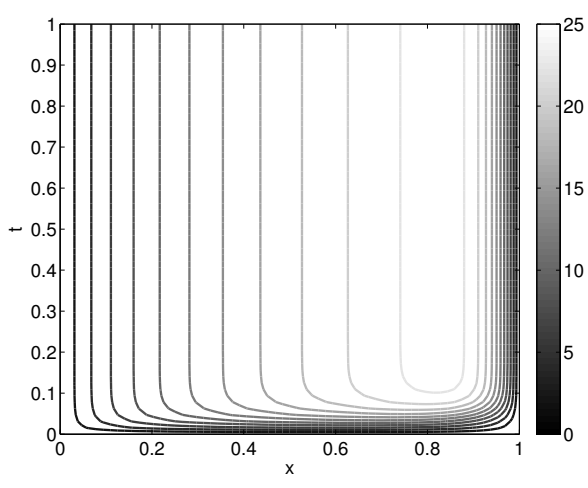

(a) Case 1: $g=1$

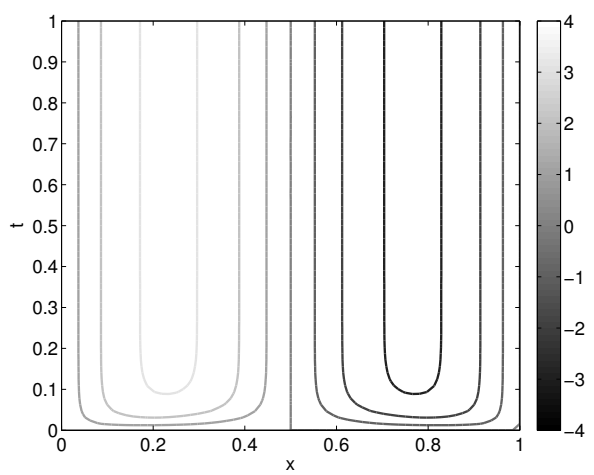

(b) Case 2: $g=\frac{1}{2}-x$

Fig. 1. The solution to the Burgers problem Case 1 and Case 2 for $\mathrm{Pe}=20$.

constant is bounded by

$$
\theta_{\delta} \equiv \sup _{w \in \mathcal{X}_{\delta}} \frac{\|w\|_{L^{2}\left(I ; L^{2}(\Omega)\right)}}{\|w\|_{\mathcal{X}_{\delta}}} \leq \sqrt{\frac{T}{3 \pi}} \approx 0.3257 \sqrt{T}
$$

as $K \rightarrow \infty$. Note that the embedding constant scales weakly with the final time $T$. For an arbitrary temporal discretization, we were unable to analytically analyze the $L^{2}-\mathcal{X}_{\delta}$ embedding constant; however, numerical experiments suggest that the constant is bounded by $\theta_{\delta} \leq 0.5773$ on any quasi-uniform temporal discretization. The $L^{4}-\mathcal{X}_{\delta}$ embedding constant can be approximated using a homotopy procedure starting from the solution to the $L^{2}-\mathcal{X}_{\delta}$ embedding problem; for related methods, see Deparis ${ }^{2}$ and Manzoni ${ }^{8}$. Numerical experiments suggest that the constant is bounded by $\rho \leq 0.81$ for any quasi-uniform space-time mesh over $\Omega=(0,1)$ and $I=(0,1]$.

\section{Numerical Results}

\subsection{Model Problems}

We consider two different forcing functions in this section. First is a constant function, $g_{1}=1$, which results in $\mathcal{F}_{1}(v ; \mu)=\mu \cdot \int_{I} \int_{\Omega} v d x d t$ with $\mu=\mathrm{Pe}^{2}$. The solution over the space-time domain for the $\mathrm{Pe}=20$ case is shown in Figure 1(a). As the Peclet number increases, the boundary layer at $x=1$ gets thinner and the initial transition time decreases. The second case uses a spatially linear source function, $g_{2}=\frac{1}{2}-x$, which results in $\mathcal{F}_{2}(v ; \mu)=\mu \cdot \int_{I} \int_{\Omega}\left(\frac{1}{2}-x\right) v d x d t$. The solution for this second case with $\mathrm{Pe}=20$ is shown in Figure 1(b). This case develops an internal layer at $x=1 / 2$, which becomes thinner as the Peclet number increases. These two cases exhibit different stability properties, as we will show shortly.

For purposes of comparison we provide here a short summary of the standard $L^{2}$ time-marching error bound developed by Nguyen et al. ${ }^{9}$ A parameter that dictates 
the effectivity of the time-marching $L^{2}$ formulation is the stability parameter $\omega^{k}$, defined as ${ }^{\mathrm{b}}$

$$
\omega^{k} \equiv \inf _{v \in V_{h}} \frac{4 b(v, u(\mu), v)+a(v, v)}{\|v\|_{L^{2}(\Omega)}}, \quad k=1, \ldots, K .
$$

In particular, a negative value of $\omega^{k}$ implies that the $L^{2}$ error estimate grows exponentially over that period of time. All results shown in this section use the exact value of $\omega^{k}$ instead of a lower bound obtained using the successive constraint method $(\mathrm{SCM})$ as done in Nguyen et al. ${ }^{9}$; i.e. we use the most favorable stability constant for the $L^{2}$ time-marching formulation.

\subsection{Stability: Small Parameter Intervals}

We will first demonstrate the improved stability of the space-time a posteriori error estimate compared to the $L^{2}$ time-marching error estimate. For the space-time formulation, we monitor the variation in the inf-sup constant, $\beta$, and the effectivity, $\Delta /\|e\|_{\mathcal{X}_{\delta}}$, with the Peclet number. For the $L^{2}$ time-marching formulation, we monitor several quantities: the minimum (normalized) stability constant, $\min _{k} \omega^{k} / \mathrm{Pe}$; the final stability constant, $\omega^{K} / \mathrm{Pe}$; the maximum effectivity, $\max _{k} \Delta^{k} /\left\|e^{k}\right\|_{L^{2}(\Omega)}$; and the final effectivity, $\Delta^{K} /\left\|e^{K}\right\|_{L^{2}(\Omega)}$.

For each case, the reduced basis approximation is obtained using the $p=2$ interpolation over a short interval of $\mathcal{D}=[\mathrm{Pe}-0.1, \mathrm{Pe}+0.1]$. Note that, the use of the short interval implies that $\tau \ll 1$, which reduces the BRR-based error bound to

$$
\Delta_{p}(\mu) \approx \frac{1}{\beta_{\mathrm{LB}}^{p}(\mu)} \epsilon_{p}
$$

In addition, as the supremizer evaluated at the centroid of the interval is close to the true supremizer over a short interval, $\beta_{\mathrm{LB}}^{p}(\mu) \approx \beta(\mu), \forall \mu \in \mathcal{D}$. In other words, we consider the short intervals to ensure a good inf-sup lower bound such that we can focus on stability independent of the quality of the inf-sup lower bound; we will later assess the effectiveness of the lower bound. The effectivity reported is the worst case value observed on 40 sampling points over the interval. ${ }^{\mathrm{c}}$

Table 1 shows the variation in the stability constant and the effectivity for Case 1 for $\mathrm{Pe}=1,10,50,100$, and 200. The stability constant for the spacetime formulation gradually decreases with Pe; accordingly, the effectivity worsens from 1.04 for $\mathrm{Pe}=1$ to 11.9 for $\mathrm{Pe}=200$. Note that the effectivity of $\mathcal{O}(10)$ is more than adequate for the purpose of reduced-order approximation as the error typically rapidly converges (i.e. exponentially) with the number of reduced basis. The $L^{2}$ time-marching formulation also performs well for this case. This is because,

\footnotetext{
${ }^{\mathrm{b}}$ In the original paper by Nguyen et al., the variable $\rho^{k}$ is used for the stability constant. Here, we use $\omega^{k}$ to avoid confusion with the $L^{4}-\mathcal{X}$ embedding constant for the space-time formulation. ${ }^{\mathrm{c}}$ The 40 sampling points are equally-spaced between $[\mathrm{Pe}-0.099, \mathrm{Pe}+0.099]$. We have found that the variation in the effectivity across the sampling point is small (less than 10\%) over the small intervals considered.
} 


\begin{tabular}{c|cc|cccc} 
& \multicolumn{2}{|c|}{ space-time } & \multicolumn{5}{|c}{$L^{2}$ time-marching } \\
$\mathrm{Pe}$ & $\beta$ & $\frac{\Delta}{\|\|_{\mathcal{X}_{\delta}}}$ & $\min _{k} \frac{\omega^{k}}{\mathrm{Pe}}$ & $\frac{\omega^{K}}{\mathrm{Pe}}$ & $\max _{k} \frac{\Delta^{k}}{\left\|e^{k}\right\|}$ & $\frac{\Delta^{K}}{\left\|e^{K}\right\|}$ \\
\hline 1 & 0.993 & 1.04 & 9.87 & 9.87 & 3.87 & 1.30 \\
10 & 0.665 & 2.23 & 0.982 & 1.32 & 3.18 & 2.11 \\
50 & 0.303 & 7.01 & 0.114 & 0.924 & 7.73 & 5.10 \\
100 & 0.213 & 9.75 & 0.0203 & 0.862 & 11.7 & 6.95 \\
200 & 0.149 & 11.9 & -0.0072 & 0.820 & 18.0 & 9.59
\end{tabular}

Table 1. Summary the inf-sup constant and effectivity for the space-time formulation and the stability constant and effectivity for the $L^{2}$ time-marching formulation for Case 1 with $g=1$.

\begin{tabular}{c|cc|cccc} 
& \multicolumn{2}{|c|}{ space-time } & \multicolumn{4}{|c}{$L^{2}$ time-marching } \\
$\mathrm{Pe}$ & $\beta$ & $\frac{\Delta}{\|e\|_{\mathcal{X}_{\delta}}}$ & $\min _{k} \frac{\omega^{k}}{\mathrm{Pe}}$ & $\frac{\omega^{K}}{\mathrm{Pe}}$ & $\max _{k} \frac{\Delta^{k}}{\left\|e^{k}\right\|}$ & $\frac{\Delta^{K}}{\left\|e^{K}\right\|}$ \\
\hline 1 & 0.999 & 1.01 & 9.84 & 9.84 & 2.80 & 2.80 \\
10 & 0.877 & 1.15 & 0.727 & 0.727 & 3.12 & 3.12 \\
20 & 0.547 & 1.84 & -0.0675 & -0.0675 & 12.4 & 12.4 \\
30 & 0.217 & 4.92 & -0.606 & -0.606 & $3.7 \times 10^{4}$ & $3.7 \times 10^{4}$ \\
50 & 0.038 & 40.8 & -1.67 & -1.67 & $6.5 \times 10^{28}$ & $6.5 \times 10^{28}$ \\
100 & 0.0077 & 259 & -4.43 & -4.43 & - & -
\end{tabular}

Table 2. Summary the inf-sup constant and effectivity for the space-time formulation and the stability constant and effectivity for the $L^{2}$ time-marching formulation for Case 2 with $g=\frac{1}{2}-x$.

even for the $\mathrm{Pe}=200$ case, the stability constant $\omega^{k} / \mathrm{Pe}$ takes on a negative value over a very short time interval and is asymptotically stable. (See Nguyen et al. ${ }^{9}$ for the detailed behavior of the stability constant over time.)

Table 2 shows the variation in the stability constant and the effectivity for Case 2 for $\mathrm{Pe}=1,10,20,50$, and 100. Note that the asymptotic stability constant for the $L^{2}$ time-marching formulation is negative for $\mathrm{Pe} \gtrsim 18.9$; consequently, the error bound grows exponentially with time even for a moderate value of the Peclet number, rendering the error bound meaningless. The stability constant for the space-time formulation is much better behaved. The effectivity of 40.8 at $\mathrm{Pe}=50$ is a significant improvement over the $10^{28}$ for the $L^{2}$ time-marching formulation, and the error estimate remains meaningful even for the $\mathrm{Pe}=100$ case.

\section{3. $N_{\mu}-p$ Interpolation over a Wide Range of Parameters}

Now we demonstrate that our certified reduced basis method provides accurate and certified solutions over a wide range of parameters using a reasonable number of snapshots. Here, we employ a simple (and rather crude) $N_{\mu}-p$ adaptive procedure to construct certified reduced basis approximations over the entire $\mathcal{D}$ with an error bound of $\Delta_{\text {tol }}=0.01$. Our $N_{\mu}-p$ approximation space is described in terms of a 
set $\mathrm{Pe}^{\text {set }}$ consisting of $N_{\mu}+1$ points that delineate the endpoints of the parameter intervals and an $N_{\mu}$-tuple $P^{\text {set }}=\left(p_{1}, \ldots, p_{N_{\mu}}\right)$ specifying the polynomial degree over each interval. Starting from a single $p=1$ interval over the entire $\mathcal{D}$, we recursively apply one of the following two operations to each interval $\left[\mathrm{Pe}_{\mathrm{L}}, \mathrm{Pe}_{\mathrm{U}}\right]=$ $\left[\mathrm{Pe}_{j}^{\text {set }}, \mathrm{Pe}_{j+1}^{\mathrm{set}}\right]$ with polynomial degree $p_{j}$ :

(a) if $\min _{\mu} \beta_{\mathrm{LB}}^{p}(\mu) \leq 0$, subdivide $\left[\mathrm{Pe}_{\mathrm{L}}, \mathrm{Pe} \mathrm{U}\right]$ into $\left[\mathrm{Pe}_{\mathrm{L}}, \mathrm{P} \mathrm{e}_{\mathrm{M}}\right] \cup\left[\mathrm{Pe}_{\mathrm{M}}, \mathrm{Pe} \mathrm{e}_{\mathrm{U}}\right]$ where $\mathrm{Pe}_{\mathrm{M}}=\left(\mathrm{Pe}_{\mathrm{L}}+\mathrm{Pe} \mathrm{e}_{\mathrm{U}}\right) / 2$, assign $p_{j}$ to both intervals, and update $\mathrm{Pe}^{\text {set }}$ and $P^{\mathrm{set}}$.

(b) if $\min _{\mu} \beta_{\mathrm{LB}}^{p}(\mu)>0$ but $\max _{\mu} \tau(\mu) \geq 1$ or $\max _{\mu} \Delta(\mu) \geq \Delta_{\text {tol }}$, then increase $p_{j}$ to $p_{j}+1$.

The operation (a) decreases the width of the parameter interval, which increases the effectiveness of the supremizer $S_{j}^{c}$ and improves the inf-sup lower bound. The operation (b) aims to decrease the residual (and hence the error) by using a higherorder interpolation, i.e. $p$-refinement. Thus, in our adaptive procedure, the $N_{\mu}$ and $p$ refinement serves two distinct purposes: improving the stability estimate and improving the approximability of the space. In particular, we assume that the solution dependence on the parameter is smooth and use (only) $p$-refinement to improve the approximability; this is in contrast to typical $h p$ adaptation where both $h$-and $p$-refinement strategies are used to improve the approximability for potentially irregular functions.

The result of applying the $N_{\mu}-p$ adaptive procedure to Case 1 is summarized in Figure 2. Here, we show variations over the parameter domain $\mathcal{D}=[1,200]$ of key quantities: a) the error and error bound; b) the error effectivity; c) the inf-sup constant and its lower bound; and d) the approximation polynomial degree. First, note that the entire parameter domain is covered using just 10 intervals consisting of 89 total interpolation points; this is despite the use of the crude adaptation process whose inefficiency is reflected in excessively accurate estimates in some of the intervals. Smaller intervals are required in the low Peclet number regimes to ensure that the normalized residual measure, $\tau$, is less than unity. The maximum error bound of $10^{-2}$ is clearly satisfied over the entire parameter range. The effectivity is of order 5 .

Table 3 shows the $p$-convergence behavior of our certified basis formulation over the final interval, $\mathcal{D}_{10}=[175.13,200.00] .{ }^{\mathrm{d}}$ Each variable is sampled at 40 equispaced sampling points over $\mathcal{D}_{10}$ and the worst case values are reported. The table confirms that the error (and the normalized residual) converges rapidly with $p$. The rapid convergence suggests that the error effectivity of $\mathcal{O}(10)$ is more than adequate, as improving the error by a factor of 10 only requires 1 or 2 additional points.

\footnotetext{
${ }^{\mathrm{d}}$ Using the $N_{\mu}-p$ adaptive procedure, this $p=8, \mathcal{D}_{10}=[175,13,200.00]$ interval is created by subdividing a $p=8, \mathcal{D}_{9}=[150.25,200.00]$ interval in the final step. This results in the use of the $p=8$ interpolant over the interval $\mathcal{D}_{10}$ in the final $N_{\mu}-p$ adapted configuration despite the error meeting the specified tolerance for $p=5$.
} 


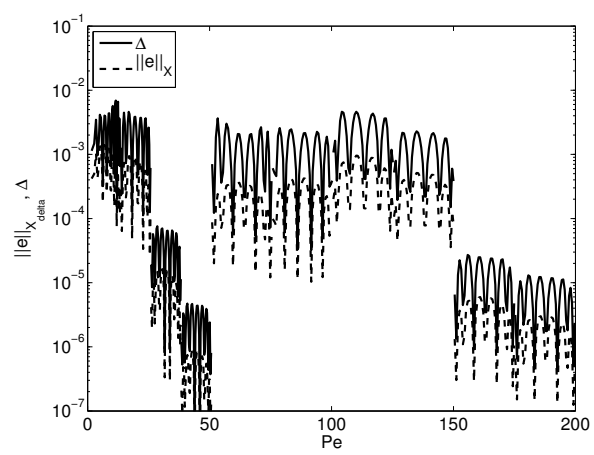

(a) error

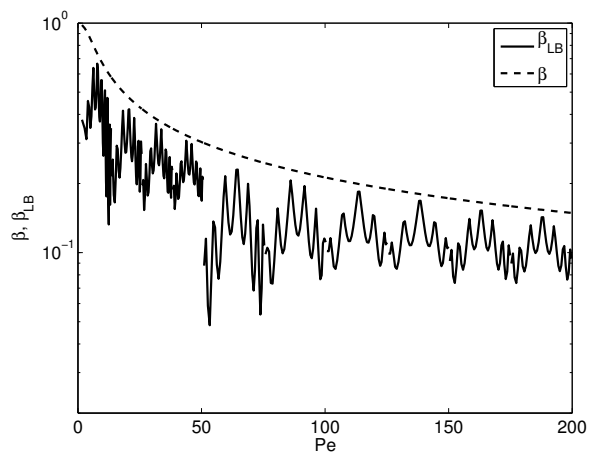

(c) inf-sup constant

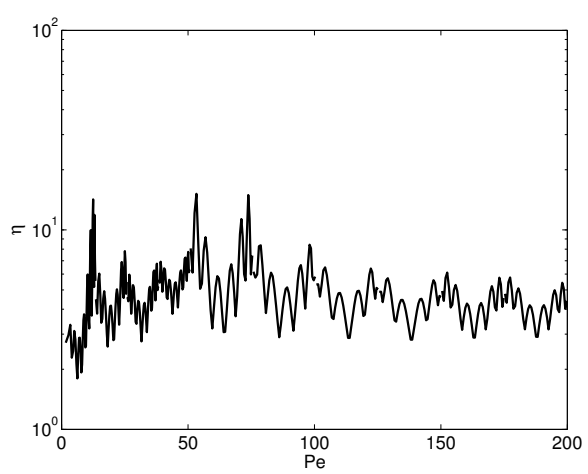

(b) error effectivity

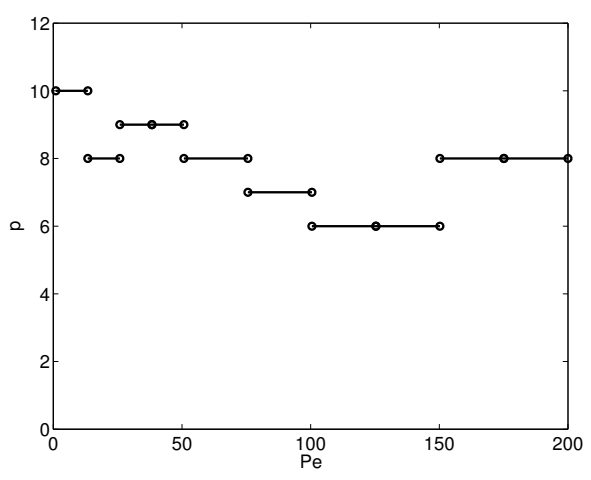

(d) $N_{\mu}-p$ selection

Fig. 2. The error, effectivity, and inf-sup constant behaviors on the final $N_{\mu}$-p adapted interpolation for Case 1.

The higher $p$ not only provides higher accuracy but also concomitantly enables construction of the BRR-based error bounds by decreasing $\tau$. Note also that the inf-sup effectivity decreases with $p$ in general as a larger number of "inf" operations are required to construct $\beta_{\mathrm{LB}}^{p}$ using the procedure introduced in Section 3.2.2.

Figure 3 shows the behavior of the error and stability constant for Case 2 over $\mathcal{D}=[1,50]$. As shown in Section 4.2, this problem is less stable than Case 1, and the classical formulation produces exponentially growing error bounds. The $N_{\mu}-p$ adaptive procedure utilizes 7 intervals consisting of 31 total interpolation points. The maximum error bound incurred over $\mathcal{D}$ is less than 0.01 . Due to the unstable nature of the problem, the effectivity worsens as the Peclet number increases. Nevertheless, unlike in the classical time-marching based formulation, our error bounds remain meaningful over the entire parameter range. For this problem, the size of the interval in the high Peclet number regime is dictated by the necessity to main a positive inf-sup lower bound. For instance, for the $p=4$ interpolation, we were unable to maintain a positive value of $\beta_{\mathrm{LB}}^{p}$ over a single interval of $[46,50]$, necessitating 


\begin{tabular}{cccccc}
$p$ & $\max _{\mu} \tau(\mu)$ & $\max _{\mu} \Delta(\mu)$ & $\max _{\mu}\|e(\mu)\|_{\mathcal{X}_{\delta}}$ & $\max _{\mu} \frac{\Delta(\mu)}{\|e(\mu)\|_{\mathcal{X}_{\delta}}}$ & $\min _{\mu} \frac{\beta_{\mathrm{LB}}(\mu)}{\beta(\mu)}$ \\
\hline 1 & $1.22 \mathrm{e}+04$ & - & $1.14 \mathrm{e}+01$ & - & 0.61 \\
2 & $2.39 \mathrm{e}+02$ & - & $6.67 \mathrm{e}-01$ & - & 0.62 \\
3 & $2.03 \mathrm{e}+01$ & - & $9.36 \mathrm{e}-02$ & - & 0.61 \\
4 & $1.38 \mathrm{e}+00$ & - & $1.11 \mathrm{e}-02$ & - & 0.61 \\
5 & $1.69 \mathrm{e}-01$ & $6.47 \mathrm{e}-03$ & $1.48 \mathrm{e}-03$ & 5.01 & 0.56 \\
6 & $2.17 \mathrm{e}-02$ & $7.69 \mathrm{e}-04$ & $1.86 \mathrm{e}-04$ & 5.05 & 0.52 \\
7 & $2.94 \mathrm{e}-03$ & $1.02 \mathrm{e}-04$ & $2.38 \mathrm{e}-05$ & 5.38 & 0.49 \\
8 & $4.13 \mathrm{e}-04$ & $1.30 \mathrm{e}-05$ & $3.00 \mathrm{e}-06$ & 5.77 & 0.47
\end{tabular}

Table 3. The $p$-convergence behavior over the final interval of Case $1, \operatorname{Pe} \in[175.13,200.00]$.

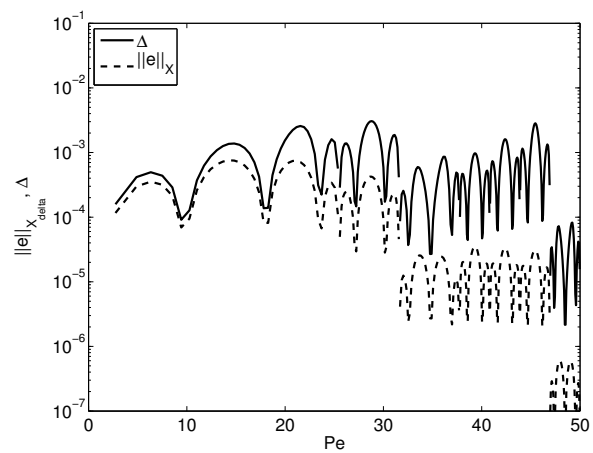

(a) error

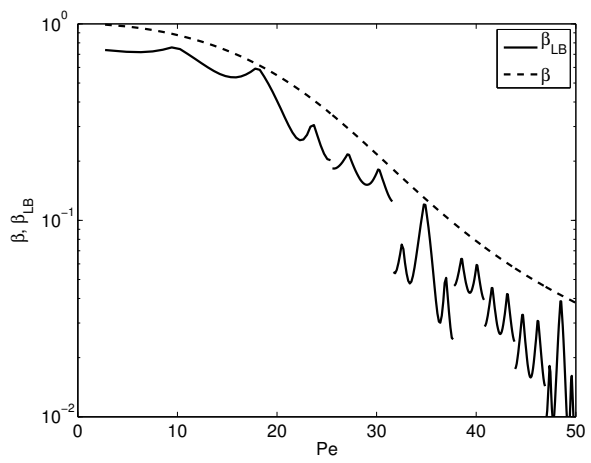

(c) inf-sup constant

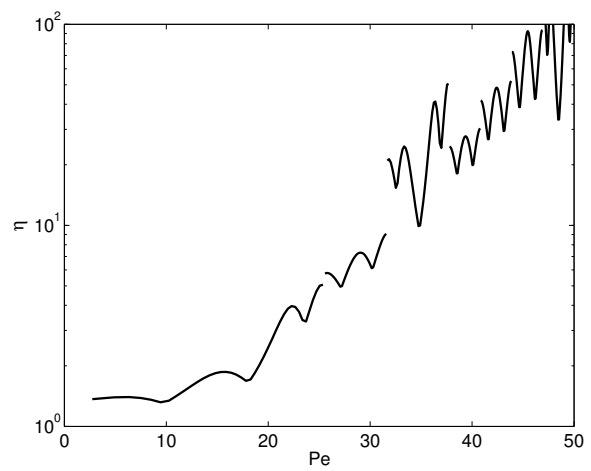

(b) error effectivity

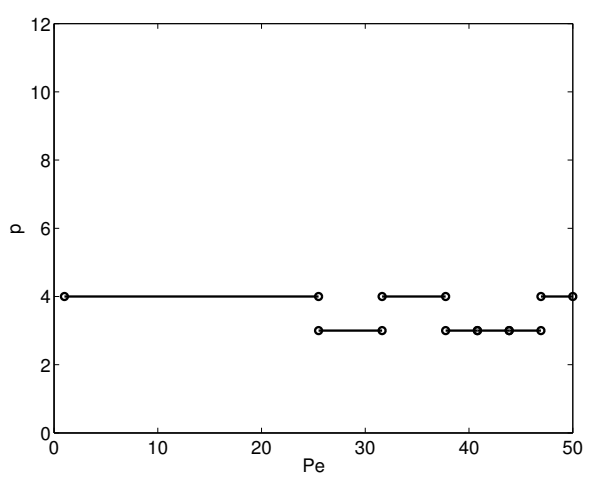

(d) $N_{\mu}-p$ selection

Fig. 3. The error, effectivity, and inf-sup constant behaviors on the final $N_{\mu}$ - $p$ adapted interpolation for Case 2.

the split into two smaller intervals. 


\begin{tabular}{cccccc}
$p$ & $\max _{\mu} \tau(\mu)$ & $\max _{\mu} \Delta(\mu)$ & $\max _{\mu}\|e(\mu)\|_{\mathcal{X}_{\delta}}$ & $\max _{\mu} \frac{\Delta(\mu)}{\|e(\mu)\|_{\mathcal{X}_{\delta}}}$ & $\min _{\mu} \frac{\beta_{\mathrm{LB}}(\mu)}{\beta(\mu)}$ \\
\hline 1 & $3.58 \mathrm{e}+03$ & - & $7.38 \mathrm{e}-02$ & - & 0.21 \\
2 & $1.39 \mathrm{e}+01$ & - & $1.03 \mathrm{e}-03$ & - & 0.22 \\
3 & $1.23 \mathrm{e}+00$ & - & $2.78 \mathrm{e}-05$ & - & 0.21 \\
4 & $2.63 \mathrm{e}-02$ & $8.22 \mathrm{e}-05$ & $6.03 \mathrm{e}-07$ & 176.78 & 0.20 \\
5 & $2.15 \mathrm{e}-02$ & $1.11 \mathrm{e}-05$ & $1.54 \mathrm{e}-08$ & 978.07 & 0.03
\end{tabular}

Table 4. The $p$-convergence behavior over the last interval of Case 2, $\mathrm{Pe} \in[46.94,50.00]$.

Table 4 shows the $p$-convergence behavior of the reduced basis formulation over $\mathcal{D}_{8}=[46.94,50]$. Similar to the previous case, the normalized residual, the error bound, and the error converge exponentially with $p$. We note that even though the worst case error effectivity is of $\mathcal{O}\left(10^{3}\right)$, the geometric mean of the effecitivies collected at the 40 sampling points is only 136 .

\section{Appendix A. Sobolev Embedding Constants}

In this appendix, we study the behavior of the $L^{4}-\mathcal{X}_{\delta}$ embedding constant required for the Brezzi-Rappaz-Raviart theory. Unfortunately, due to the nonlinearity, we have not been able to analyze the $L^{4}-\mathcal{X}_{\delta}$ problem analytically. To gain some insight into the behavior of the embedding constant using analytical techniques, let us consider two closely related linear problems, $L^{2}-\mathcal{X}$ embedding and $L^{2}-\mathcal{X}_{\delta}$ embedding, in Appendix A.1 and A.2. Then, we will numerical investigate the behavior of the $L^{4}-\mathcal{X}_{\delta}$ embedding constant in Appendix A.3. ${ }^{\mathrm{e}}$

\section{A.1. $L^{2}-\mathcal{X}$ Embedding}

Let us first consider $L^{2}-\mathcal{X}$ embedding. The embedding constant is defined by

$$
\theta \equiv \sup _{w \in \mathcal{X}} \frac{\|w\|_{L^{2}\left(I ; L^{2}(\Omega)\right)}}{\|w\|_{\mathcal{X}}}
$$

which is obtained by solving a (linear) eigenproblem

$$
\begin{aligned}
(w, v)_{\mathcal{X}}-\lambda(w, v) & =0, \quad \forall v \in \mathcal{X} \\
1-\|w\|_{L^{2}\left(I ; L^{2}(\Omega)\right)}^{2} & =0
\end{aligned}
$$

and setting $\theta=\lambda_{\min }^{-1 / 2}$. Applying the Fourier decomposition in the spatial domain, ${ }^{\mathrm{f}}$ we can express the eigenproblem as: find eigenpairs $\left(w^{k_{x}}, \lambda^{k_{x}}\right) \in H_{0}^{1}(I) \times \mathbb{R}$ such

eAnalysis in this appendix is "formal"; for brevity, some of the assumptions or arguments required related to completeness or compactness may be omitted.

${ }^{\mathrm{f}}$ We could directly analyze the spatial discretization with appropriate modification of the $k_{x}$ Fourier symbol per the usual von Neumann analysis. Here we consider a continuous-in-space case for simplicity. 
that

$$
\begin{array}{r}
\frac{1}{k_{x}^{2} \pi^{2}} \int_{I} \dot{v}^{k_{x}}(t) \dot{w}^{k_{x}}(t) d t+k_{x}^{2} \pi^{2} \int_{I} v^{k_{x}}(t) w^{k_{x}}(t)=\lambda^{k_{x}} \int_{I} v^{k_{x}}(t) w^{k_{x}}(t) d t, \\
\forall v^{k_{x}} \in H_{0}^{1}(I),
\end{array}
$$

where $v^{k_{x}} \in H_{0}^{1}(I)$ is the temporally-varying Fourier coefficient associated with the $k_{x}$-mode and $H_{0}^{1}(I) \equiv\left\{v \in C_{0}(I): v(t=0)=0\right\}$. It is straightforward to show that the eigenmodes of the continuous problem are given by

$$
v^{k_{x}, k_{t}}(t)=\sin \left(\pi\left(k_{t}-\frac{1}{2}\right) \frac{t}{T}\right), \quad k_{t}=1,2, \ldots
$$

and the corresponding eigenvalues are

$$
\lambda^{k_{x}, k_{t}}=k_{x}^{2} \pi^{2}+\frac{1}{k_{x}^{2} T^{2}}\left(k_{t}-\frac{1}{2}\right)^{2} .
$$

The expression clearly shows that the minimum eigenvalue is achieved for $k_{t}=1$ for all $T$. In particular, for $T>1 /(4 \pi)$, the minimum eigenvalue corresponds to $k_{x}=k_{t}=1$, and its value is

$$
\lambda_{\min }=\frac{1}{4 T^{2}}+\pi^{2} .
$$

Because $\mathcal{X}_{\delta} \subset \mathcal{X}$ for any temporal discretization of $I$, we have

$$
\hat{\lambda}_{\min } \equiv \inf _{w \in \mathcal{X}_{\delta}} \frac{\|w\|_{\mathcal{X}}}{\|w\|_{L^{2}\left(I ; L^{2}(\Omega)\right)}} \geq \inf _{w \in \mathcal{X}} \frac{\|w\|_{\mathcal{X}}}{\|w\|_{L^{2}\left(I ; L^{2}(\Omega)\right)}}=\lambda_{\min }=\frac{1}{4 T^{2}}+\pi^{2}
$$

for all $T>1 /(4 \pi)$. (Appropriate bounding constant may be deduced from the expression for the eigenvalues of the continuous problem even for $T<1 /(4 \pi)$.) In other words, for any $\mathcal{X}_{\delta} \subset \mathcal{X}$, the $L^{2}-\mathcal{X}$ embedding constant is bounded by

$$
\theta \leq\left(\frac{1}{4 T^{2}}+\pi^{2}\right)^{-1 / 2}
$$

for $T>1 /(4 \pi)$. Note that this bounding constant is not significantly different from that for the standard $L^{2}-H_{(0)}^{1}$ embedding problem, $\theta_{L^{2}-H_{(0)}^{1}}=\left(\pi^{2} /\left(4 T^{2}\right)+\pi^{2}\right)^{-1 / 2}$.

\section{A.2. $L^{2}-\mathcal{X}_{\delta}$ Embedding}

Now let us consider $L^{2}-\mathcal{X}_{\delta}$ embedding. The embedding constant is defined by

$$
\theta_{\delta} \equiv \sup _{w \in \mathcal{X}_{\delta}} \frac{\|w\|_{L^{2}\left(I ; L^{2}(\Omega)\right)}}{\|w\|_{\mathcal{X}_{\delta}}}
$$

where we recall that $\|w\|_{\mathcal{X}_{\delta}}^{2}=\|\dot{w}\|_{L^{2}\left(I ; V^{\prime}\right)}^{2}+\|\bar{w}\|_{L^{2}(I ; V)}^{2}$. Similar to the $L^{2}-\mathcal{X}$ embedding problem, the solution is given by finding the minimum eigenvalue of

$$
\begin{aligned}
(w, v)_{\mathcal{X}_{\delta}}-\lambda(w, v) & =0, \quad \forall v \in \mathcal{X}_{\delta} \\
1-\|w\|_{L^{2}\left(I ; L^{2}(\Omega)\right.}^{2} & =0,
\end{aligned}
$$


and setting $\theta_{\delta}=\lambda_{\min }^{-1 / 2}$. However, as the $\mathcal{X}_{\delta}$ norm is dependent on the temporal mesh by construction, we must consider temporally discrete spaces for our analysis. Let $V_{\Delta t} \subset H_{0}^{1}(I)$ be the piecewise linear temporal approximation space. Then, the Fourier decomposition in the spatial domain results in an eigenproblem: find eigenpairs $\left(w_{\delta}^{k_{x}}, \lambda^{k_{x}}\right) \in V_{\Delta t} \times \mathbb{R}$ such that

$$
\begin{array}{r}
\frac{1}{k_{x}^{2} \pi^{2}} \int_{I} \dot{v}^{k_{x}}(t) \dot{w}^{k_{x}}(t) d t+k_{x}^{2} \pi^{2} \int_{I} \bar{v}^{k_{x}}(t) \bar{w}^{k_{x}}(t)=\lambda^{k_{x}} \int_{I} v^{k_{x}}(t) w^{k_{x}}(t) d t, \\
\forall v^{k_{x}} \in V_{\Delta t},
\end{array}
$$

where $\bar{v}^{k_{x}}$ over the $I^{k}$ is given by $\left(\Delta t^{k}\right)^{-1} \int_{I^{k}} v^{k_{x}} d x$.

For $V_{\Delta t}$ with a constant time step (i.e. $\Delta t=\Delta t^{1}=\cdots=\Delta t^{K}$ ), the $k$-th entry of the $k_{t}$-th eigenmode $\mathbf{v}^{k_{x}, k_{t}} \in \mathbb{R}^{K}$ is given by

$$
\left(\mathbf{v}^{k_{x}, k_{t}}\right)_{k}=\sin \left(\pi\left(k_{t}-\frac{1}{2}\right) \frac{k}{K}\right) \text {. }
$$

Accordingly, the eigenvalues may be expressed as

$$
\lambda^{k_{x}, k_{t}}(K ; T)=\frac{\frac{K}{k_{x}^{2} \pi^{2} T}\left(1-\cos \left(\pi\left(k_{t}-\frac{1}{2}\right) \frac{1}{K}\right)\right)+\frac{k_{x}^{2} \pi^{2} T}{4 K}\left(1+\cos \left(\pi\left(k_{t}-\frac{1}{2}\right) \frac{1}{K}\right)\right)}{\frac{T}{6 K}\left(2+\cos \left(\pi\left(k_{t}-\frac{1}{2}\right) \frac{1}{K}\right)\right)} .
$$

To estimate the minimum eigenvalue, we first relax the restriction that $k_{x}$ be an integer; with the relaxation, the minimizing value of $k_{x}, k_{x}^{*}$, is given by

$$
k_{x}^{*}\left(k_{t}\right)=\frac{4^{1 / 2}}{\pi} \sqrt{\frac{K}{T}}\left(\tan \left(\frac{\pi}{2}\left(\frac{k_{t}}{K}-\frac{1}{2 K}\right)\right)\right)^{1 / 2} .
$$

Furthermore, for $k_{x}=k_{x}^{*}$, it can be shown that the eigenvalue is minimized for $k_{t}=K$. Thus, for any given final time $T$ and the number of time steps $K$, a lower bound (due to the continuous relaxation on $k_{x}$ ) of eigenvalues can be expressed as

$$
\lambda_{\mathrm{LB}}(K ; T)=\min _{k_{t} \in 1, \ldots, K} \min _{k_{x} \in \mathbb{R}^{+}} \lambda^{k_{x}, k_{t}}(K ; T)=\frac{6 K}{T} \frac{\sin \left(\pi\left(1-\frac{1}{2 K}\right)\right)}{2+\cos \left(\pi\left(1-\frac{1}{2 K}\right)\right)} .
$$

In the limit of $K \rightarrow \infty$, the eigenvalue approaches

$$
\lim _{K \rightarrow \infty} \lambda_{\mathrm{LB}}(K ; T)=\frac{3 \pi}{T} .
$$

Thus, in the limit of $K \rightarrow \infty$, the $L^{2}-\mathcal{X}_{\delta}$ embedding constant for $V_{\Delta t}$ with a constant time step is given by $\theta_{\delta}=\sqrt{\frac{T}{3 \pi}} \approx 0.3257 \sqrt{T}$. Note that the embedding constant scales weakly with the final time $T$. We also note that the optimal spatial wave number behaves like $k_{x}^{*} \rightarrow \infty$ as $K \rightarrow \infty$.

Unfortunately, for $V_{\Delta t}$ with non-constant time stepping, we cannot deduce the embedding constant analytically. Here, we numerically demonstrate that the $L^{2}$ - $\mathcal{X}_{\delta}$ embedding constant is indeed bounded for all quasi-uniform meshes. In particular, we compute the embedding constant on temporal meshes characterized by the number of elements, $K$, and a logarithmic mesh grading factor, $q$, where $q=0$ 


\begin{tabular}{c|cccccccccc} 
& \multicolumn{10}{c}{ mesh grading factor, $q$} \\
$K$ & -2 & -1 & 0 & 1 & 2 & 3 & 4 & 5 & 7 & 10 \\
\hline 2 & 0.3483 & 0.3379 & 0.3903 & 0.4783 & 0.5156 & 0.5550 & 0.5683 & 0.5760 & 0.5771 & 0.5773 \\
4 & 0.3344 & 0.3252 & 0.3423 & 0.4623 & 0.5379 & 0.5692 & 0.5758 & 0.5771 & 0.5769 & 0.5760 \\
8 & 0.3224 & 0.3164 & 0.3298 & 0.4629 & 0.5422 & 0.5696 & 0.5761 & 0.5772 & 0.5772 & 0.5771 \\
16 & 0.3159 & 0.3148 & 0.3267 & 0.4635 & 0.5419 & 0.5692 & 0.5759 & 0.5772 & 0.5771 & 0.5772 \\
32 & 0.3147 & 0.3145 & 0.3259 & 0.4636 & 0.5418 & 0.5691 & 0.5758 & 0.5771 & 0.5773 & 0.5773 \\
64 & 0.3144 & 0.3144 & 0.3257 & 0.4636 & 0.5418 & 0.5691 & 0.5758 & 0.5771 & 0.5773 & 0.5773 \\
128 & 0.3144 & 0.3144 & 0.3258 & 0.4636 & 0.5418 & 0.5691 & 0.5758 & 0.5771 & 0.5773 & 0.5773
\end{tabular}

Table 5. The variation in the $L^{2}-\mathcal{X}$ embedding constant with the number of time intervals, $K$, and the mesh grading factor, $q$, for $T=1$.

corresponds to a uniform mesh, $q>0$ implies that elements are clustered toward $t=0$. For $q$ sufficiently large, the first temporal element is of order $\Delta t^{1} \approx 10^{-q} T$. Without loss of generality, we pick $T=1$.

The result of the calculation is summarized in Table 5. First, the table confirms that, on a uniform temporal mesh $(q=0)$, the embedding constant converges to the analytical value of $(3 \pi)^{-1 / 2} \approx 0.3257$ as $K$ increases. The embedding constant increases with the mesh grading factor, $q$; however, the constant appears to asymptote to 0.5773 as $q \rightarrow \infty$. Thus, the numerical result suggests that the $L^{2}-\mathcal{X}_{\delta}$ is bounded for all quasi-uniform meshes by 0.5773 .

\section{A.3. $L^{4}-\mathcal{X}_{\delta}$ Embedding}

Recall that the $L^{4}-\mathcal{X}_{\delta}$ embedding constant is defined as

$$
\rho \equiv \sup _{w \in \mathcal{X}} \frac{\|w\|_{L^{4}\left(I ; L^{4}(\Omega)\right)}}{\|w\|_{\mathcal{X}_{\delta}}}
$$

To find the embedding constant we solve a nonlinear eigenproblem

$$
\begin{aligned}
(w, v)_{\mathcal{X}}-\lambda\left(w^{3}, v\right) & =0, \quad \forall v \in \mathcal{X} \\
1-\|w\|_{L^{4}\left(I ; L^{4}(\Omega)\right.}^{4} & =0
\end{aligned}
$$

and set $\rho=\lambda_{\min }^{-1 / 2}$. This nonlinear eigenproblem is solved using a homotopy procedure. Namely, we successively solve a family of problems,

$$
\begin{aligned}
(w, v)_{\mathcal{X}}-\lambda\left((1-\alpha)\left(w^{2}, v\right)+\alpha\left(w^{3}, v\right)\right) & =0, \quad \forall v \in \mathcal{X} \\
1-\left((1-\alpha)\|w\|_{L^{2}\left(I ; L^{2}(\Omega)\right)}^{2}+\alpha\|w\|_{L^{4}\left(I ; L^{4}(\Omega)\right)}^{4}\right) & =0,
\end{aligned}
$$

starting from $\alpha=0$, which corresponds to $L^{2}-\mathcal{X}_{\delta}$ embedding, and slowly increase the value of $\alpha$ until $\alpha=1$, which corresponds to $L^{4}-\mathcal{X}_{\delta}$ embedding.

The numerical values of the embedding constant on different meshes is shown in Table 6. Similar to the $L^{2}-\mathcal{X}_{\delta}$ embedding constant, the $L^{4}-\mathcal{X}_{\delta}$ embedding constant increases with the number of temporal time steps, $K$, and the mesh grading factor, 


\begin{tabular}{c|ccccccc} 
& \multicolumn{7}{|c}{ mesh grading factor, $q$} \\
$K$ & -2 & -1 & 0 & 1 & 2 & 3 & 4 \\
\hline 2 & 0.4508 & 0.4308 & 0.5716 & 0.6479 & 0.6694 & 0.7211 & 0.7389 \\
4 & 0.4333 & 0.4479 & 0.4955 & 0.6367 & 0.7227 & 0.7454 & 0.7520 \\
8 & 0.4475 & 0.4962 & 0.4824 & 0.6242 & 0.7188 & 0.7537 & 0.7567 \\
16 & 0.4958 & 0.4956 & 0.4791 & 0.6315 & 0.7174 & 0.7496 & 0.7626 \\
32 & 0.4955 & 0.4955 & 0.4774 & 0.6295 & 0.7371 & 0.8084 & - \\
64 & 0.4955 & 0.4955 & 0.4755 & 0.6283 & 0.7462 & 0.7808 & -
\end{tabular}

Table 6 . The variation in the $L^{4}-\mathcal{X}$ embedding constant with the number of time intervals, $K$, and the mesh grading factor, $q$, for $T=1$.

q. Again, the embedding constant appears to be bounded. Based on the table, we approximate the $L^{4}-\mathcal{X}_{\delta}$ embedding constant for any quasi-uniform mesh to be bounded by $\rho=0.81$.

\section{Appendix B. Comparison of Inf-Sup Lower Bound Construction Procedures}

This appendix details the relationship between the inf-sup lower bound constructed using the procedure developed in Section 3.2.2 and the natural-norm Successive Constraint Method (SCM) method. ${ }^{6}$ For convenience, we refer to our method based on the explicit calculation of the lower and upper bounds of the correction factors as "LU" and that based on the Successive Constraint Method as "SCM." Both LU and SCM procedures are based on the decomposition ${ }^{\mathrm{g}}$

$$
\begin{aligned}
\beta(\mu) & \equiv \inf _{w \in \mathcal{X}} \sup _{v \in \mathcal{Y}} \frac{\partial \mathcal{G}\left(w, \tilde{u}^{p}, v\right)}{\|w\|_{\mathcal{X}}\|v\|_{\mathcal{Y}}} \geq \inf _{w \in \mathcal{X}} \frac{\partial \mathcal{G}\left(w, \tilde{u}^{p}, S^{c} w\right)}{\|w\|_{\mathcal{X}}\left\|S^{c} w\right\|} \\
& \geq \underbrace{\left.\inf _{w \in \mathcal{X}} \frac{\left\|S^{c} w\right\|_{\mathcal{Y}}}{\|w\|_{\mathcal{X}}}\right)}_{\beta^{c}} \cdot \underbrace{\left(\inf _{w \in \mathcal{X}} \frac{\partial \mathcal{G}\left(w, \tilde{u}^{p}, S^{c} w\right)}{\left\|S^{c} w\right\|_{\mathcal{Y}}^{2}}\right)}_{\hat{\beta}^{c}(\mu)},
\end{aligned}
$$

where we have identified the inf-sup constant evaluated at the centroid by $\beta^{c}$ and the correction factor by $\hat{\beta}^{c}(\mu)$. Note that the correction factor may be expressed as

$$
\begin{aligned}
\hat{\beta}^{c}(\mu) & =\inf _{w \in \mathcal{X}} \frac{\partial \mathcal{G}\left(w, \tilde{u}^{p}, S^{c} w\right)}{\left\|S^{c} w\right\|_{\mathcal{Y}}^{2}}=\sum_{k=1}^{p+1} \inf _{w \in \mathcal{X}} \psi_{k}^{p}(\mu) \frac{\partial \mathcal{G}\left(w, u_{k}, S^{c} w\right)}{\left\|S^{c} w\right\|_{\mathcal{Y}}^{2}} \\
& =\sum_{k=1}^{p+1} \inf _{w \in \mathcal{X}} \psi_{k}^{p}(\mu) \frac{\left(S^{k} w, u_{k}, S^{c} w\right)_{\mathcal{Y}}}{\left\|S^{c} w\right\|_{\mathcal{Y}}^{2}} .
\end{aligned}
$$

Our LU method and SCM differ in the way they construct bounds of $\hat{\beta}^{c}(\mu)$.

${ }^{\mathrm{g}}$ The subscript $j$ on the supremizer $S_{j}^{c}$ (and later solution snapshots $u_{j, k}$ ) that denotes the domain number is suppressed in this appendix for notational simplicity. 
Let us recast our LU formulation as a linear programming problem, the language in which the SCM is described. We compute a lower bound of the correction factor, $\hat{\beta}_{\mathrm{LB}, \mathrm{LU}}^{c}(\mu) \leq \hat{\beta}^{c}(\mu), \forall \mu \in \mathcal{D}_{j}$, by first constructing a box in $\mathbb{R}^{p+1}$ that encapsulates the lower and upper bounds of contribution of each term of the correction factor, i.e.

$$
B_{\mathrm{LU}}=\prod_{k=1}^{p+1}\left[\inf _{w \in \mathcal{X}} \frac{\left(S^{k} w, u_{k}, S^{c} w\right)_{\mathcal{Y}}}{\left\|S^{c} w\right\|_{\mathcal{Y}}^{2}}, \sup _{w \in \mathcal{X}} \frac{\left(S^{k} w, u_{k}, S^{c} w\right)_{\mathcal{Y}}}{\left\|S^{c} w\right\|_{\mathcal{Y}}^{2}}\right] .
$$

Then, we solve a (rather simple) linear programming problem

$$
\hat{\beta}_{\mathrm{LB}, \mathrm{UL}}^{c}(\mu)=\inf _{y \in B^{\mathrm{UL}}} \sum_{k=1}^{p+1} \psi_{k}^{p}(\mu) y_{k},
$$

the solution to which is given by choosing either extrema for each coordinate of the bounding box $B_{\mathrm{LU}}$ based on the sign of $\psi_{k}^{p}(\mu)$, as explicitly stated in Section 3.2.2.

Let us now consider a special case of SCM where the SCM sampling points are the interpolation points, $\mu^{k}, k=1, \ldots, p+1$, of the $N_{\mu}$ - $p$ interpolation scheme. The SCM bounding box is given by

$$
B_{\mathrm{SCM}}=\prod_{k=1}^{p+1}\left[-\frac{\gamma_{k}}{\beta^{c}}, \frac{\gamma_{k}}{\beta^{c}}\right]
$$

where

$$
\gamma_{k} \equiv \sup _{w \in \mathcal{X}} \frac{\left\|S^{k} w\right\|_{\mathcal{Y}}}{\|w\|_{\mathcal{X}}}
$$

Since the kernel of $B_{\mathrm{LU}}$ is bounded by

$$
\left|\frac{\left(S^{k} w, u_{k}, S^{c} w\right)_{\mathcal{Y}}}{\left\|S^{c} w\right\|_{\mathcal{Y}}^{2}}\right| \leq \frac{\left\|S^{k} w\right\|_{\mathcal{Y}}}{\|w\|_{\mathcal{X}}} \frac{\|w\|_{\mathcal{X}}}{\left\|S^{c} w\right\|_{\mathcal{Y}}} \leq \sup _{w \in \mathcal{X}} \frac{\left\|S^{k} w\right\|_{\mathcal{Y}}}{\|w\|_{\mathcal{X}}}\left(\inf _{w \in \mathcal{}} \frac{\left\|S^{c} w\right\|_{\mathcal{Y}}}{\|w\|_{\mathcal{X}}}\right)^{-1}=\frac{\gamma_{k}}{\beta^{c}},
$$

for $k=1, \ldots, p+1$, we have

$$
B_{\mathrm{LU}} \subset B_{\mathrm{SCM}} .
$$

Furthermore, as the SCM sampling points correspond to the interpolation points, the SCM linear programming constraints

$$
\sum_{k=1}^{p+1} \psi_{k}^{p}\left(\mu_{l}\right) y_{k} \geq \bar{\beta}^{c}\left(\mu_{l}\right), \quad l=1, \ldots, p+1
$$

simplify to (using $\left.\psi_{k}^{p}\left(\mu_{l}\right)=\delta_{k l}\right)$

$$
y_{k} \geq \bar{\beta}^{c}\left(\mu_{k}\right), \quad k=1, \ldots, p+1,
$$

where

$$
\bar{\beta}^{c}\left(\mu_{k}\right)=\inf _{w \in \mathcal{X}} \frac{\left(S^{k} w, u_{k}, S^{c} w\right)_{\mathcal{Y}}}{\left\|S^{c} w\right\|_{\mathcal{Y}}^{2}} .
$$


We recognize that the this constraint is in fact identical to the lower bound box constraint of $B_{\mathrm{LU}}$. Thus, the space over which the SCM lower bound is computed,

$$
D_{\mathrm{SCM}}^{\mathrm{LB}}=\left\{y \in B^{\mathrm{SCM}}: y_{k} \geq \bar{\beta}^{c}\left(\mu_{k}\right), \quad k=1, \ldots, p+1\right\},
$$

satisfies

$$
B_{\mathrm{LU}} \subset D_{\mathrm{SCM}}^{\mathrm{LB}} .
$$

More specifically, $D_{\mathrm{SCM}}^{\mathrm{LB}}$ has the same lower bounds as $B_{\mathrm{LU}}$ but has loser upper bounds than $B_{\mathrm{LU}}$. Consequently, we have

$$
\inf _{y \in D_{\mathrm{SCM}}^{\mathrm{LB}}} \sum_{k=1}^{p+1} \psi_{k}^{p}(\mu) y_{k}=\hat{\beta}_{\mathrm{LB}, \mathrm{SCM}}^{c}(\mu) \leq \hat{\beta}_{\mathrm{LB}, \mathrm{LU}}^{c}(\mu)=\inf _{y \in B^{\mathrm{UL}}} \sum_{k=1}^{p+1} \psi_{k}^{p}(\mu) y_{k} \leq \hat{\beta}^{c} .
$$

Thus, if the SCM sampling points are the same as the interpolation points of the $N_{\mu}-p$ interpolation scheme, then our LU formulation gives a tighter inf-sup lower bound than SCM.

\section{Acknowledgments}

This work was supported by OSD/AFOSR/MURI Grant FA9550-09-1-0613, ONR Grant N00014-11-1-0713, and the Deutsche Forschungsgemeinschaft (DFG) uder Ur-63/9 and GrK1100.

\section{References}

1. F. Brezzi, J. Rappaz, and P. A. Raviart. Finite dimensional approximation of nonlinear problems. part I: Branches of nonsingular solutions. Numer. Math., 36:1-25, 1980.

2. S. Deparis. Reduced basis error bound computation of parameter-dependent NavierStokes equations by the natural norm approach. SIAM J. Numer. Anal., 46(4):20392067, 2008.

3. J. L. Eftang, A. T. Patera, and E. M. Rønquist. An " $h p "$ certified reduced basis method for parametrized elliptic partial differential equations. SIAM J. Sci. Comput., 32(6):3170-3200, 2010.

4. M. A. Grepl and A. T. Patera. A posteriori error bounds for reduced-basis approximations of parametrized parabolic partial differential equations. Math. Model. Numer. Anal., 39(1):157-181, 2005.

5. B. Haasdonk and M. Ohlberger. Reduced basis method for finite volume approximations of parametrized linear evolution equations. Math. Model. Numer. Anal., 42(2):277-302, 2008.

6. D. B. P. Huynh, D. J. Knezevic, Y. Chen, J. S. Hesthaven, and A. T. Patera. A naturalnorm successive constraint method for inf-sup lower bounds. Comput. Methods Appl. Mech. Engrg., 199:1963-1975, 2010.

7. D. J. Knezevic, N.-C. Nguyen, and A. T. Patera. Reduced basis approximation and $a$ posteriori error estimation for the parametrized unsteady Boussinesq equations. Math. Mod. Meth. Appl. S., 21(7):1415-1442, 2011.

8. A. Manzoni. Reduced models for optimal control, shape optimization and inverse problems in haemodynamics. PhD thesis, École Polytechnique Fédérale de Lausanne, 2012. 
9. N.-C. Nguyen, G. Rozza, and A. T. Patera. Reduced basis approximation and a posteriori error estimation for the time-dependent viscous Burgers' equation. Calcolo, 46:157-185, 2009.

10. A. Quarteroni and A. Valli. Numerical Approximation of Partial Differential Equations. Springer, New York, 1997.

11. C. Schwab and R. Stevenson. Space-time adaptive wavelet methods for parabolic evolution problems. Math. Comp., 78(267):1293-1318, 2009.

12. K. Urban and A. T. Patera. An improved error bound for reduced basis approximation of linear parabolic problems. Math. Comp., page submitted, 2012.

13. K. Urban and A. T. Patera. A new error bound for reduced basis approximation of parabolic partial differential equations. C. R. Acad. Sci. Paris, Ser. I, 350:203-207, 2012.

14. K. Veroy and A. T. Patera. Certified real-time solution of the parametrized steady incompressible Navier-Stokes equations: rigorous reduced-basis a posteriori error bounds. Internat. J. Numer. Methods Fluids, 47:773-788, 2005. 